The ASTROPhysical Journal, 247:403-418, 1981 July 15

(C) 1981. The American Astronomical Society. All rights reserved. Printed in U.S.A.

\title{
EMISSION-LINE PROFILES AND KINEMATICS OF THE NARROW-LINE REGION IN SEYFERT AND RADIO GALAXIES
}

\author{
Timothy M. Heckman, ${ }^{1}$ George K. Miley, ${ }^{1}$ and Wil J. M. van Breugel ${ }^{1}$ \\ Sterrewacht Leiden \\ AND \\ HARVEY R. BUTCHER \\ Kitt Peak National Observatory ${ }^{2}$ \\ Received 1980 September 29; accepted 1981 January 28
}

\begin{abstract}
We report measurements of the profiles of the $[\mathrm{O} \mathrm{II}] \lambda 5007$ narrow emission lines in 36 Seyfert and radio galaxies. These observations were obtained using the High-Gain Video Spectrometer on the $4 \mathrm{~m}$ telescope at Kitt Peak National Observatory with relatively high spectral resolution $(\sim 2.2$ $\AA=130 \mathrm{~km} \mathrm{~s}^{-1}$ ). Although the main purpose of the observations was to study the emission-line profiles, spatial information along a $2^{\prime \prime} \times 166^{\prime \prime}$ slit was also obtained.

Parameters describing the widths and asymmetries of the spatially integrated [O III] $\lambda 5007$ profiles are defined and tabulated for each galaxy. Several interesting effects are shown to be present in our sample as a whole.

1. The majority of the profiles are markedly asymmetric, exhibiting a sharper falloff to the red than to the blue. This is in the opposite sense from the asymmetry that has been previously noted in the broad Balmer emission lines.

2. The velocities derived from the narrow lines are systematically negative compared with the systemic velocities of the surrounding galaxies.

3. There is an excellent correlation between the degree of asymmetry of the lines and the $\mathrm{H} \alpha / \mathrm{H} \beta$ ratio.

4. A good correlation also exists between the degree of asymmetry and the $[\mathrm{O} \mathrm{II}] /[\mathrm{O} \mathrm{III}]$ and $[\mathrm{O} \mathrm{I}] /[\mathrm{O} \mathrm{III}]$ ratios for galaxies that have broad permitted-line components.

5. The relation between the line widths and absolute luminosities of the steep-spectrum, kiloparsec-sized, nuclear radio sources in Seyfert 2 galaxies is confirmed and extended to Seyfert 1 and radio galaxies.

Three objects with spatially resolved emission-line regions, as well as some other galaxies with interesting line profiles, are discussed individually.

The implications of our results for the kinematics, ionization state, and energetics of active nuclei are considered. The data suggest that the narrow emission-line region consists of gas and dust flowing radially (probably outward) with respect to the nucleus. It is shown that the presence of dust is probably affecting the efficiency of photoionization from the central nuclear source in class 1 Seyferts, while the lack of corresponding evidence in the class 2 Seyferts may require an alternative source of ionization. The nature of the relationship between the thermal plasma of the emission-line gas and the relativistic plasma of the radio source is discussed briefly. Finally, evidence is presented that the kinematics and physical conditions of the narrow-line emitting gas are not strongly coupled.
\end{abstract}

Subject headings: galaxies: internal motions - galaxies: nuclei - galaxies: Seyfert

\section{INTRODUCTION}

Emission lines in active nuclei are of two types: "broad-line" with velocity widths of a few thousand

${ }^{1}$ Visiting Astronomer, Kitt Peak National Observatory.

${ }^{2}$ Operated by the Association of Universities for Research in Astronomy, Inc., under contract with the National Science Foundation. kilometers per second, and "narrow-line" with widths of a few hundred kilometers per second (Weedman 1977). The lines are believed to be produced in two quite different zones. The broad-line region (BLR) has a characteristic size of smaller than a parsec, whereas the narrow-line region (NLR) has dimensions of a few kiloparsecs. In this article we are mainly concerned with the narrow lines. 
There have been many studies of the relative intensities of the narrow lines (e.g., Baldwin 1975; Osterbrock, Koski, and Phillips 1976; Costero and Osterbrock 1977; Osterbrock 1977; Grandi and Osterbrock 1978; Koski 1978; Penston and Fosbury 1978), but the conclusions that can be drawn from such studies are often very model-dependent. Except for a few individual cases (e.g., Glaspey et al. 1976; Glaspey, Walker, and Stockton 1976; Pelat and Alloin 1980), comparatively little work has been carried out on the line profiles. This is probably because relatively high spectral resolution $\left(\ll 500 \mathrm{~km} \mathrm{~s}^{-1}\right.$ ) is required to sufficiently resolve the narrow lines. Since a study of the profiles can provide a powerful and direct method for probing the kinematics of active nuclei, we have begun a program to measure narrow-line profiles for a large sample of them.

This paper presents results for the [O III] $\lambda 5007$ line in 36 Seyfert and radio galaxies, based on observations having a spectral resolution of $\sim 2.2 \AA\left(\sim 130 \mathrm{~km} \mathrm{~s}^{-1}\right)$. The observational procedure and reduction are described in § II. In § III some parameters which describe the profiles are extracted and tabulated. The distributions of these parameters and their relationship to other parameters of the galaxies in our sample are considered in § IV. Comments on individual interesting profiles are made in $\S \mathrm{V}$, including three galaxies in which the emission-line region is significantly resolved spatially. Section VI is a brief attempt to discuss the consequences of our results for the energetics of active galaxies. Finally, § VII summarizes the principal conclusions of the article and suggests some directions for future work.

\section{OBSERVATIONS AND DATA REDUCTION}

The data were obtained during 1979 November and 1980 May using the High-Gain Video Spectrometer (HGVS) together with the R-C Spectrograph on the $4 \mathrm{~m}$ Mayall telescope at Kitt Peak National Observatory. The HGVS consists of an RCA silicon intensified target (SIT) which is lens-coupled to the output phosphor of a high-gain, magnetically focused Carnegie image tube. It is a two-dimensional detector with 128 pixels in the spatial direction and 512 spectral channels.

In the configuration used the separation between pixels corresponds to $\sim 1^{\prime \prime} .3$ and $0.67 \AA$, respectively, and the spectral resolution corresponding to FWHM intensity was $\sim 2.2 \AA$ or $130 \mathrm{~km} \mathrm{~s}^{-1}$ at [O III] $\lambda 5007$ using a $2^{\prime \prime}$ slit width. The slit position angle used was somewhat arbitrary, and was chosen to take into account both the position of interesting known extranuclear features and the efficiency of the observing schedule.
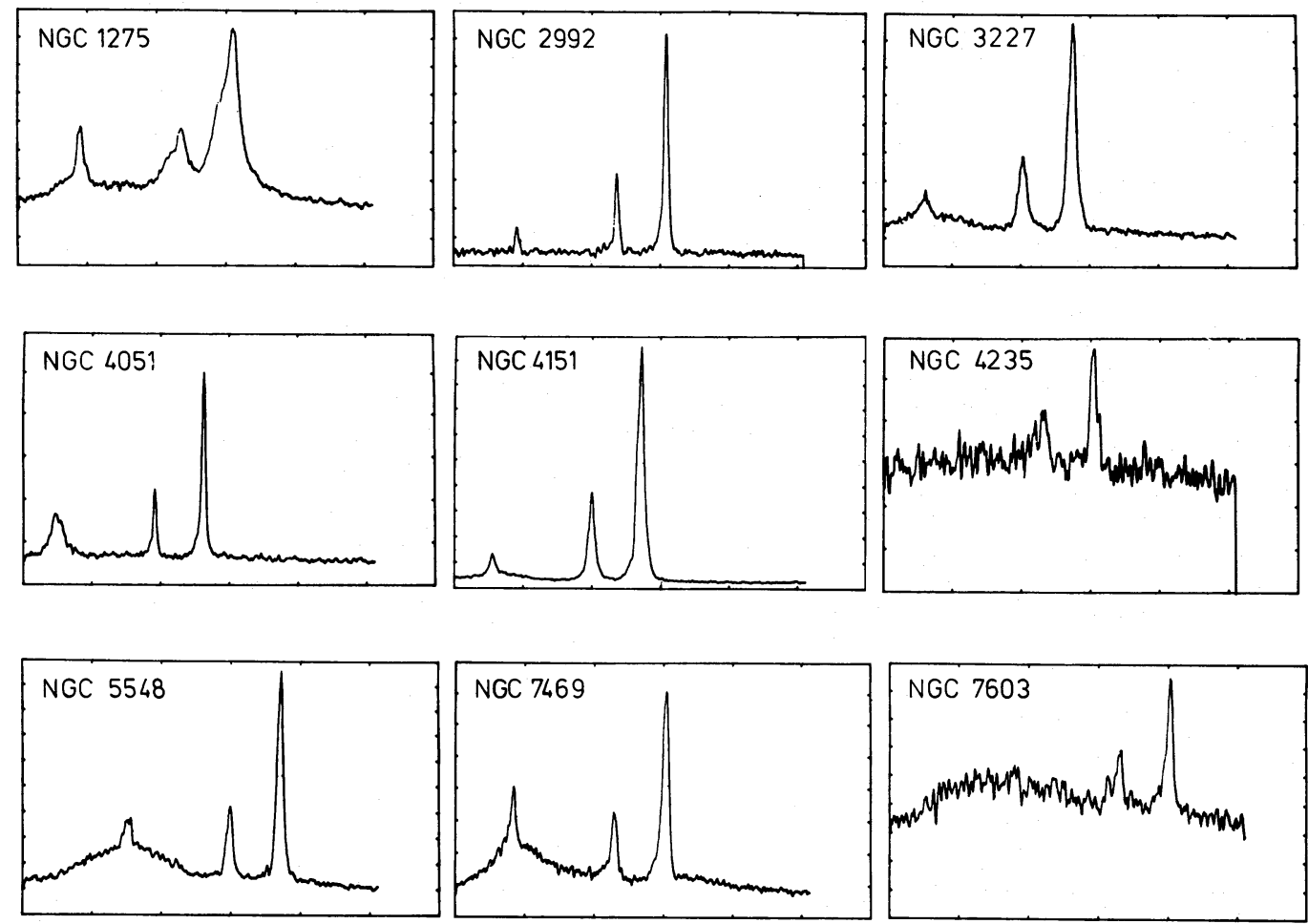

FIG. $1 a$

FIG. 1.- Spectra of 36 active nuclei in our program plotted as counts per second in arbitrary units. The three lines that are visible are $\mathrm{H} \beta$ $\lambda 4861,[\mathrm{O} \mathrm{III}] \lambda 4959$, and [O $\mathrm{III}] \lambda 5007$, the latter being the strongest. The $x$-axes are linear wavelength scales. The corresponding velocities are listed in Table 1 . 

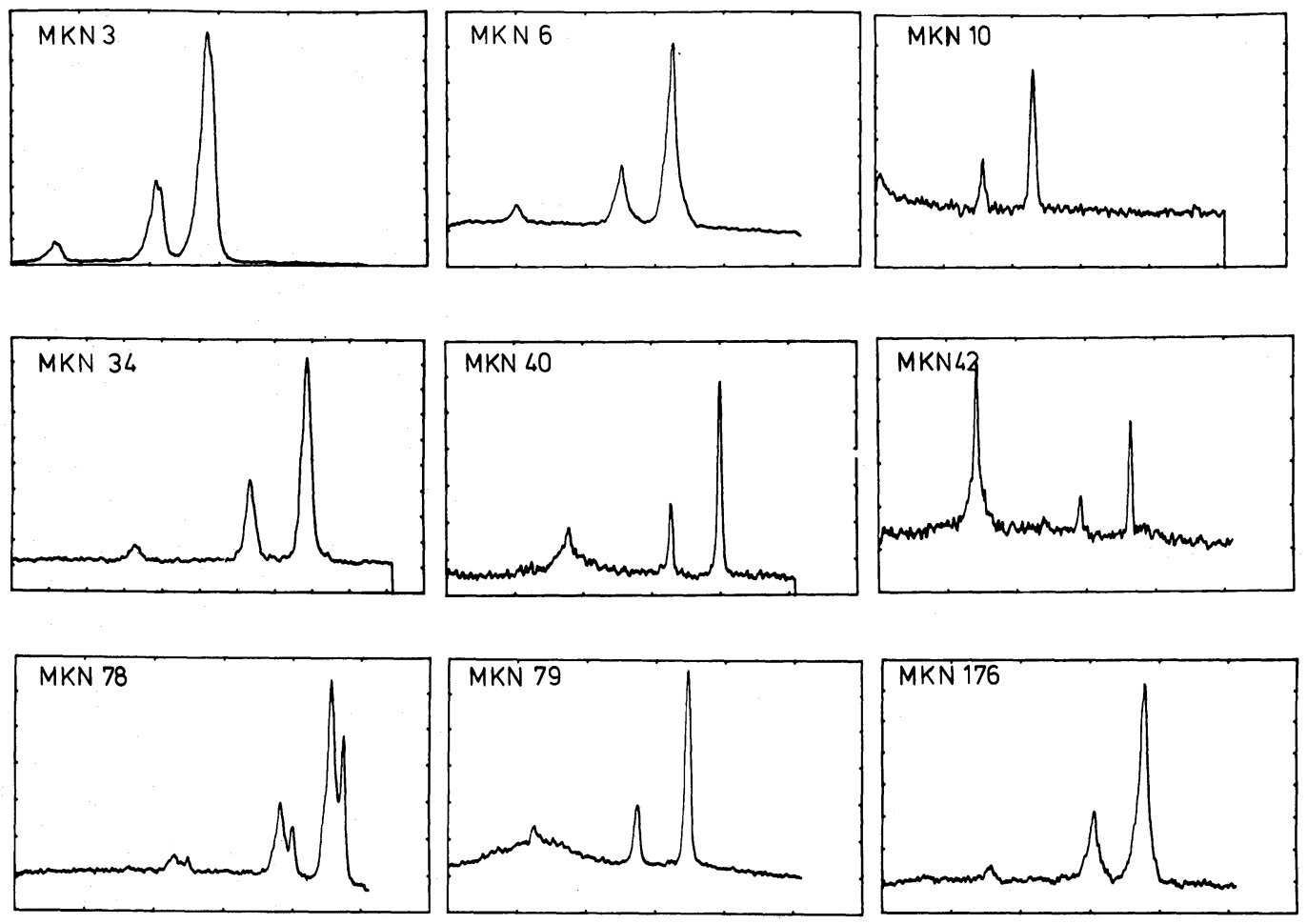

FIG. $1 b$
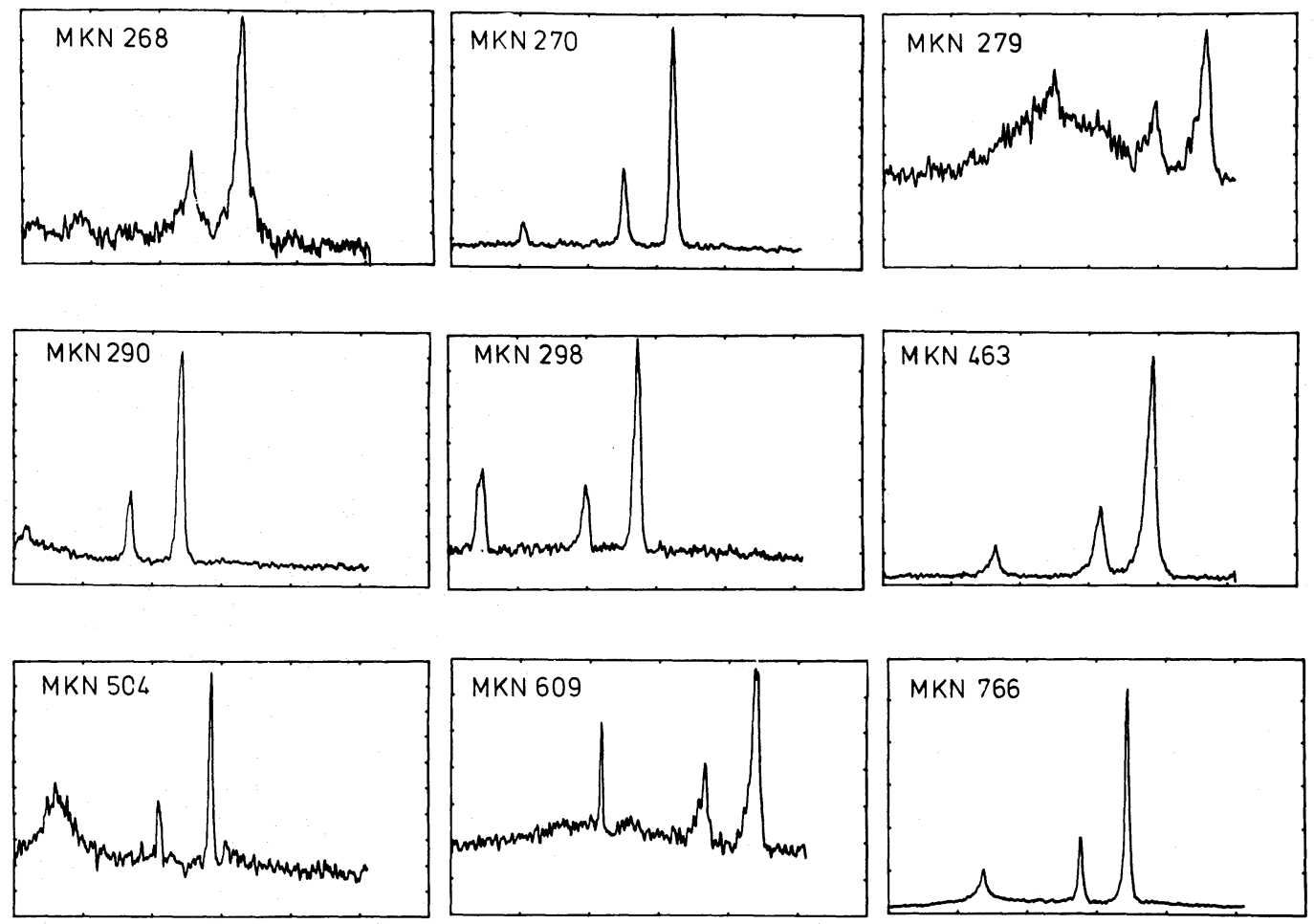

FIG. $1 c$ 

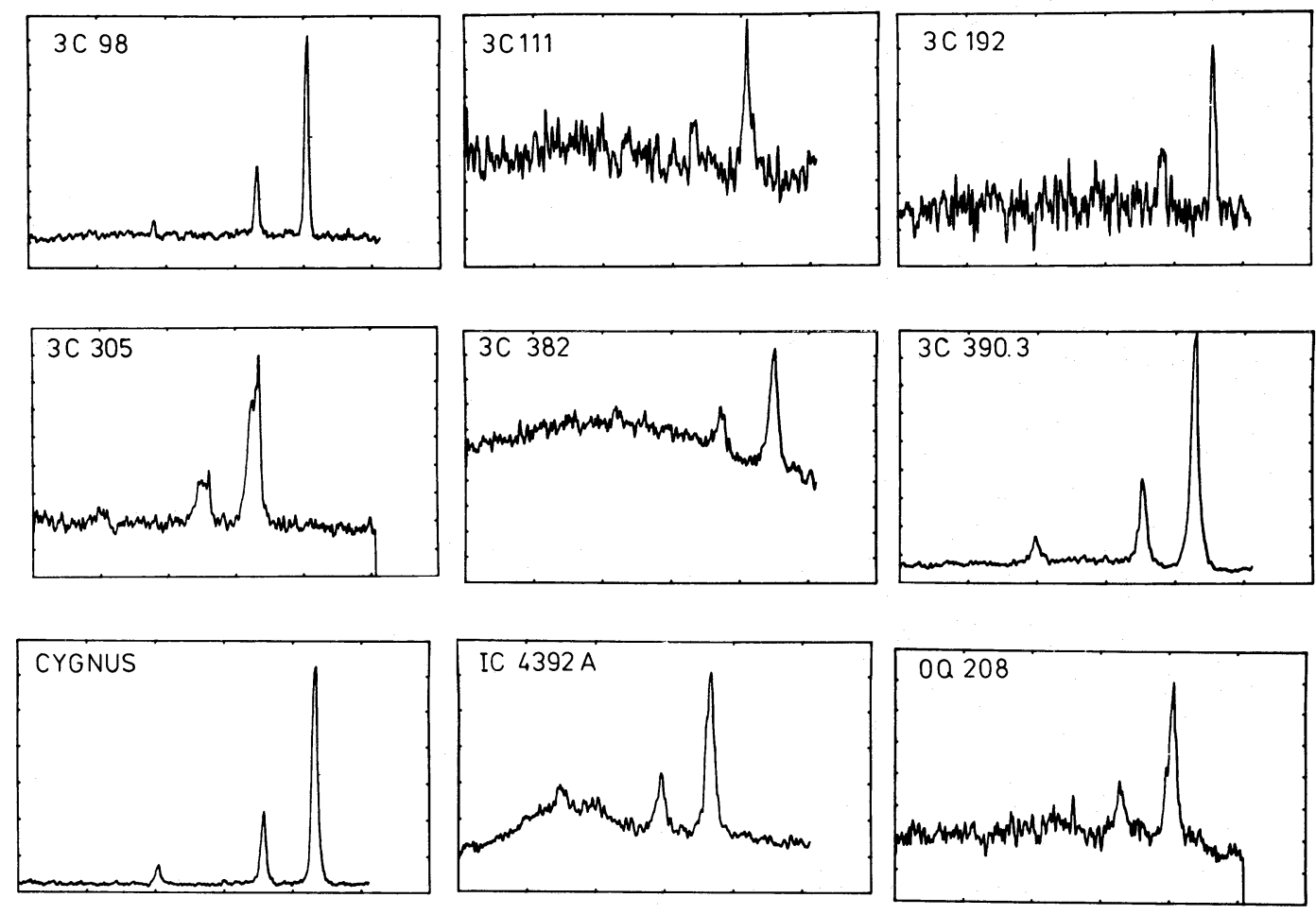

FIG. $1 d$

During much of the observations, the seeing was poor, varying from $\sim 3^{\prime \prime}$ to $\sim 5^{\prime \prime}$.

The integration time spent on each nucleus was usually 13 minutes, except for several of the weaker objects for which two 13 minute integrations were averaged.

The data were corrected for variations in the pixel-topixel detector sensitivity using observations of a quartz lamp. The smooth variation in sensitivity along the slit direction was corrected using observations of blank sky to define a "slit function." Since significant variations in detector sensitivity along the wavelength direction do not occur across an individual line profile, no attempt was made to calibrate the intensity as a function of wavelength.

He-Ne-Ar comparison spectra were obtained while tracking each galaxy either before or after an integration. These were used individually to remove $S$ distortion and line curvature in the corresponding galaxy spectrum.

For those spectra in which the sky was present at a significant level, its effect was digitally subtracted from the data using the "galaxy-free" portion of the spectrum along the slit to generate a template of the sky.

The final spectra were reduced to one-dimensional form by averaging over several spatial pixel rows. The resultant spectra are displayed in Figure 1. Since only a handful of galaxies have lines which are significantly resolved spatially along the slit (see $\S \mathrm{V}$ ), the profiles shown in Figure 1 are believed to be generally representative of the global line profile of the NLR.

\section{LINE PROFILE PARAMETERS}

The galaxies in our sample are listed in column (1) of Table 1 in order of increasing NGC, Markarian (Mrk), and $3 \mathrm{C}$ numbers, and concluding with several miscellaneous galaxies. Their class is given in column (2) with Seyfert 1 and 2 having the designations S1 and S2, respectively, and broad-line and narrow-line radio galaxies being denoted as BRG and NRG, respectively. The position angle at which the $2^{\prime \prime} \times 166^{\prime \prime}$ slit was oriented is given in column (3) as well as the length along the slit that was used to produce the spectra in Figure 1 .

For each [O III] $\lambda 5007$ profile, we have measured the full line width at levels of $80 \%, 50 \%$, and $20 \%$ of the peak intensity above the continuum. These widths (W80, W50, W20) are listed in columns (4)-(6), respectively, after deconvolution of the instrumental profile as determined from the He-Ne-Ar comparison lines.

Uncertainties in the widths were estimated by comparison of the [O III] $\lambda 5007$ and [O III] $\lambda 4959$ profiles, which should be identical except for a scale factor set by atomic physics. The lower signal-to-noise ratio in the $\lambda 4959$ profile was accounted for in these estimates.

Columns (7)-(9) contain the velocities of the line centers at three respective levels (C80, C50, C20). Each 
TABLE 1

[O III] $\lambda 5007$ Emission Line Profile Parameters

\begin{tabular}{|c|c|c|c|c|c|c|c|c|c|c|}
\hline \multirow{2}{*}{\multicolumn{2}{|c|}{$\begin{array}{c}\text { Galaxy } \\
\text { (1) }\end{array}$}} & \multirow{2}{*}{$\begin{array}{l}\text { Class } \\
\text { (2) }\end{array}$} & \multirow{2}{*}{$\begin{array}{c}\text { PA/Length } \\
(0 / 1) \\
(3)\end{array}$} & \multirow{2}{*}{\multicolumn{2}{|c|}{$\frac{\text { W80 }}{(4) \quad\left(\mathrm{km} \mathrm{s} \mathrm{s}^{-1}\right)}$}} & \multirow{2}{*}{$\frac{W 20}{(6)}$} & \multirow{2}{*}{$\begin{array}{l}\mathrm{c} 80 \\
(7)\end{array}$} & \multirow{2}{*}{$\frac{\mathrm{C} 50}{\left(\mathrm{~km} \mathrm{~s} \mathrm{~s}^{-1}\right.}$} & \multirow{2}{*}{$\frac{c 20}{(9)}$} & \multirow{2}{*}{$\begin{array}{l}\mathrm{AI}_{20} \\
(10)\end{array}$} \\
\hline & & & & & & & & & & \\
\hline $\begin{array}{l}\text { JGC } \\
\text { JGC } \\
\text { JGC } \\
\text { JGC } \\
\text { JGC } \\
\text { JGC } \\
\text { JGC } \\
\text { JGC } \\
\text { JGC }\end{array}$ & $\begin{array}{l}1275 \ldots \ldots \\
2992 \ldots \ldots \\
3227 \ldots \ldots \\
4051 \ldots \ldots \\
4151 \ldots \ldots \\
4235 \ldots \ldots \\
5548 \ldots \ldots \\
7469 \ldots \ldots \\
7603 \ldots \ldots\end{array}$ & $\begin{array}{l}\text { NRG } \\
\text { S2 } \\
\text { S1 } \\
\text { S1 } \\
\text { S1 } \\
\text { S1 } \\
\text { S1 } \\
\text { S1 } \\
\text { S1 }\end{array}$ & $\begin{array}{c}90 / 12 \\
120 / 17 \\
45 / 12 \\
45 / 12 \\
45 / 6 \cdot 5 \\
120 / 6.5 \\
135 / 6.5 \\
90 / 12 \\
90 / 9\end{array}$ & $\begin{array}{l}460 \\
140 \\
260 \\
110 \\
190 \\
270 \\
190 \\
240 \\
170\end{array}$ & $\begin{array}{l}1360 \pm 10 \\
260 \pm 15 \\
540 \pm 10 \\
210 \pm 10 \\
460 \pm 5 \\
400 \pm 40 \\
380 \pm 10 \\
390 \pm 10 \\
330 \pm 25\end{array}$ & $\begin{array}{l}450 \\
900 \\
420 \\
760 \\
710 \\
640 \\
670 \\
670\end{array}$ & $\begin{array}{r}245 \\
-10 \\
50 \\
20 \\
32 \\
0 \\
15 \\
10 \\
5\end{array}$ & $\begin{array}{r}4920 \\
2375 \\
-- \\
595 \\
905 \\
2220 \\
5030 \\
4790 \\
8785\end{array}$ & $\begin{array}{r}-80 \\
-15 \\
5 \\
-35 \\
20 \\
80 \\
-45 \\
-60 \\
-105\end{array}$ & $\begin{array}{r}0.27 \pm 0.01 \\
0.03 \pm 0.06 \\
0.10 \pm 0.03 \\
0.29 \pm 0.05 \\
0.03 \pm 0.10 \\
-0.23 \pm 0.12 \\
0.20 \pm 0.03 \\
0.21 \pm 0.04 \\
0.330 .07\end{array}$ \\
\hline $\begin{array}{l}\text { Mrk } \\
\text { Mrk } \\
\text { Mrk } \\
\text { Mrk } \\
\text { Mrk } \\
\text { Mrk } \\
\text { Mrk } \\
M r k \\
\text { Mrk } \\
\text { Mrk } \\
\text { Mrk } \\
\text { Mrk } \\
\text { Mrk } \\
\text { Mrk } \\
\text { Mrk } \\
\text { Mrk } \\
\text { Mrk } \\
\text { Mrk }\end{array}$ & $\begin{array}{l}3 \ldots \ldots \ldots \\
6 \ldots \ldots \ldots \\
10 \ldots \ldots \\
34 \ldots \ldots \\
40 \ldots \ldots \\
42 \ldots \ldots \\
78 \ldots \ldots \ldots \\
79 \ldots \ldots \\
176 \ldots \ldots \\
268 \ldots \ldots \\
270 \ldots \ldots \\
279 \ldots \ldots \\
290 \ldots \ldots \\
298 \ldots \ldots \\
463 \ldots \ldots \\
504 \ldots \ldots \\
609 \ldots \ldots \\
766 \ldots \ldots\end{array}$ & $\begin{array}{l}\mathrm{S} 2 \\
\mathrm{~S} 1 \\
\mathrm{~S} 1 \\
\mathrm{~S} 2 \\
\mathrm{~S} 1 \\
\mathrm{~S} 1 \\
\mathrm{~S} 2 \\
\mathrm{~S} 1 \\
\mathrm{~S} 2 \\
\mathrm{~S} 2 \\
\mathrm{~S} 2 \\
\mathrm{~S} 1 \\
\mathrm{~S} 1 \\
\mathrm{~S} 2 \\
\mathrm{~S} 2 \\
\mathrm{~S} 1 \\
\mathrm{~S} 2 \\
\mathrm{~S} 1\end{array}$ & $\begin{array}{c}153 / 6.5 \\
153 / 6.5 \\
45 / 12 \\
45 / 6.5 \\
155 / 6.5 \\
153 / 12 \\
153 / 6.5 \\
153 / 14 \\
153 / 12 \\
45 / 12 \\
45 / 9 \\
45 / 6.5 \\
45 / 6.5 \\
100 / 6.5 \\
45 / 14 \\
45 / 6.5 \\
90 / 12 \\
45 / 12\end{array}$ & $\begin{array}{l}495 \\
240 \\
160 \\
250 \\
130 \\
100 \\
870^{a} \\
210 \\
260 \\
310 \\
180 \\
280 \\
240 \\
210 \\
220 \\
90 \\
290 \\
120\end{array}$ & $\begin{array}{l}850 \pm 5 \\
560 \pm 10 \\
360 \pm 20 \\
570 \pm 10 \\
220 \pm 10 \\
160 \pm 20 \\
1030 \pm 10 \\
360 \pm 10 \\
490 \pm 10 \\
530 \pm 30 \\
380 \pm 10 \\
580 \pm 30 \\
380 \pm 10 \\
410 \pm 15 \\
540 \pm 10 \\
170 \pm 20 \\
450 \pm 25 \\
240 \pm 10\end{array}$ & $\begin{array}{r}1230 \\
520 \\
820 \\
360 \\
260 \\
1440 \\
530 \\
1100 \\
1070 \\
600 \\
1470 \\
530 \\
620 \\
980 \\
290 \\
940 \\
480\end{array}$ & $\begin{array}{r}50 \\
65 \\
-5 \\
25 \\
5 \\
10 \\
0^{a} \\
-10 \\
20 \\
20 \\
-30 \\
20 \\
10 \\
35 \\
50 \\
0 \\
5 \\
15\end{array}$ & $\begin{array}{r}4050 \\
5680 \\
-- \\
15270 \\
6175 \\
7395 \\
11285 \\
6635 \\
7930 \\
-- \\
3125 \\
9070 \\
13115 \\
10285 \\
15040 \\
10790 \\
10225 \\
3868\end{array}$ & $\begin{array}{r}-95 \\
0 \\
5 \\
0 \\
-20 \\
-25 \\
-105 \\
-15 \\
-80 \\
-50 \\
15 \\
-310 \\
-10 \\
-40 \\
-80 \\
0 \\
-100 \\
-20\end{array}$ & $\begin{array}{r}0.22 \pm 0.01 \\
0.10 \pm 0.01 \\
-0.03 \pm 0.07 \\
0.04 \pm 0.03 \\
0.15 \pm 0.06 \\
0.30 \pm 0.13 \\
0.20 \pm 0.01 \\
0.02 \pm 0.04 \\
0.18 \pm 0.02 \\
0.12 \pm 0.06 \\
-0.16 \pm 0.04 \\
0.44 \pm 0.04 \\
0.09 \pm 0.04 \\
0.25 \pm 0.04 \\
0.26 \pm 0.02 \\
0.00 \pm 0.12 \\
0.22 \pm 0.05 \\
0.17 \pm 0.04\end{array}$ \\
\hline $\begin{array}{l}3 \mathrm{C} \\
3 \mathrm{C} \\
3 \mathrm{C} \\
3 \mathrm{C} \\
3 \mathrm{C} \\
3 \mathrm{C}\end{array}$ & $\begin{array}{l}98 \ldots \ldots \ldots \\
111 \ldots \ldots \\
192 \ldots \ldots \\
305 \ldots \ldots \\
382 \ldots \ldots \\
390.3^{6} \ldots \ldots\end{array}$ & $\begin{array}{l}\text { NRG } \\
\text { BRG } \\
\text { NRG } \\
\text { NRG } \\
\text { BRG } \\
\text { BRG }\end{array}$ & $\begin{array}{c}170 / 12 \\
153 / 6.5 \\
45 / 4 \\
57 / 14 \\
45 / 6.5 \\
150 / 3.9\end{array}$ & $\begin{array}{r}130 \\
70 \\
190 \\
370 \\
220 \\
250\end{array}$ & $\begin{array}{l}230 \pm 15 \\
260 \pm 35 \\
330 \pm 35 \\
740 \pm 15 \\
490 \pm 25 \\
410 \pm 10\end{array}$ & $\begin{array}{r}370 \\
750 \\
420 \\
1090 \\
830 \\
780\end{array}$ & $\begin{array}{r}10 \\
5 \\
-25 \\
50 \\
0 \\
10\end{array}$ & $\begin{array}{r}9020 \\
14710 \\
17805 \\
12445 \\
17370 \\
16640\end{array}$ & $\begin{array}{r}-10 \\
40 \\
15 \\
-100 \\
-20 \\
-5\end{array}$ & $\begin{array}{r}-0.10 \pm 0.10 \\
-0.20 \pm 0.15 \\
0.26 \pm 0.03 \\
0.05 \pm 0.06 \\
0.03 \pm 0.02\end{array}$ \\
\hline $\begin{array}{l}\text { Cyg } \\
\text { OQ } \\
\text { IC }\end{array}$ & $\begin{array}{r}A \ldots \ldots \ldots \\
208 \ldots \ldots \ldots \\
4329 \text { A. . }\end{array}$ & $\begin{array}{ll}\text { - } & \text { NRG } \\
\text { - } & \text { BRG } \\
\text { Sl }\end{array}$ & $\begin{array}{l}150 / 6 \cdot 5 \\
155 / 6 \cdot 5 \\
135 / 4\end{array}$ & $\begin{array}{l}200 \\
180 \\
230\end{array}$ & & & $\begin{array}{r}10 \\
-10 \\
25\end{array}$ & $\begin{array}{r}16795 \\
22970 \\
4780\end{array}$ & $\begin{array}{r}5 \\
-60 \\
55\end{array}$ & $\begin{array}{r}0.01 \pm 0.03 \\
0.12 \pm 0.06 \\
-0.08 \pm 0.05\end{array}$ \\
\hline
\end{tabular}

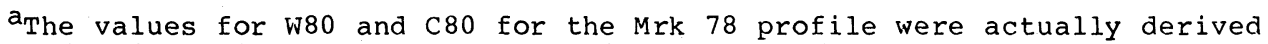
at the $\sim 67 \%$ intensity level because of its unusual double-peaked shape.

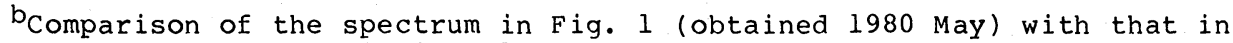
Osterbrock, Koski, and Phillips (1976) shows that the broad HB component has faded appreciably relative to the narrow [O III] and $H \beta$ lines. A spectrum of $\mathrm{H} \alpha$, also obtained during the 1980 May observations, shows the same effect. The notch in the broad $\mathrm{H} \alpha$ profile, seen in the Osterbrock et al. spectrum, now nearly descends to the level of the continuum. Further monitoring of the profiles of the broad lines in this object would be worthwhile.

is defined by the unweighted average line center (Fig. 2 illustrates the definitions of widths and centers in a schematic way). The corresponding velocities listed in columns (7)-(9) are all heliocentric and are defined as $\mathrm{C}\left(\Delta \lambda / \Delta \lambda_{0}\right)$. From the degree of internal consistency of the dispersion solutions, they have typical uncertainties of $\sim 30 \mathrm{~km} \mathrm{~s}^{-1}$.

In addition we have estimated the degree of asymmetry in the profile at the $20 \%$ intensity level by measuring the half widths to the left and right of the line center defined at the $80 \%$ intensity level (WL20 and WR20).
As a measure of the asymmetry, we have defined an asymmetry index $\mathrm{AI}_{20}=\mathrm{WL} 20-\mathrm{WR} 20 / \mathrm{WL} 20+\mathrm{WR} 20$ $=\Delta \mathrm{W} 20 / \mathrm{W} 20$. Values of $\mathrm{AI}_{20}$ with the instrumental spectral resolution deconvolved are listed in column (10) of Table 1. As with the line widths, the uncertainties in $\mathrm{AI}_{20}$ have been estimated by comparison of the $\lambda 4959$ and $\lambda 5007$ [O III] lines, taking appropriate account of the higher signal-to-noise ratio in the latter lines.

Since SIT tubes can have beam-pulling effects (Schechter and Gunn 1979) that could cause wavelength shifts and asymmetries in emission lines, we have care- 


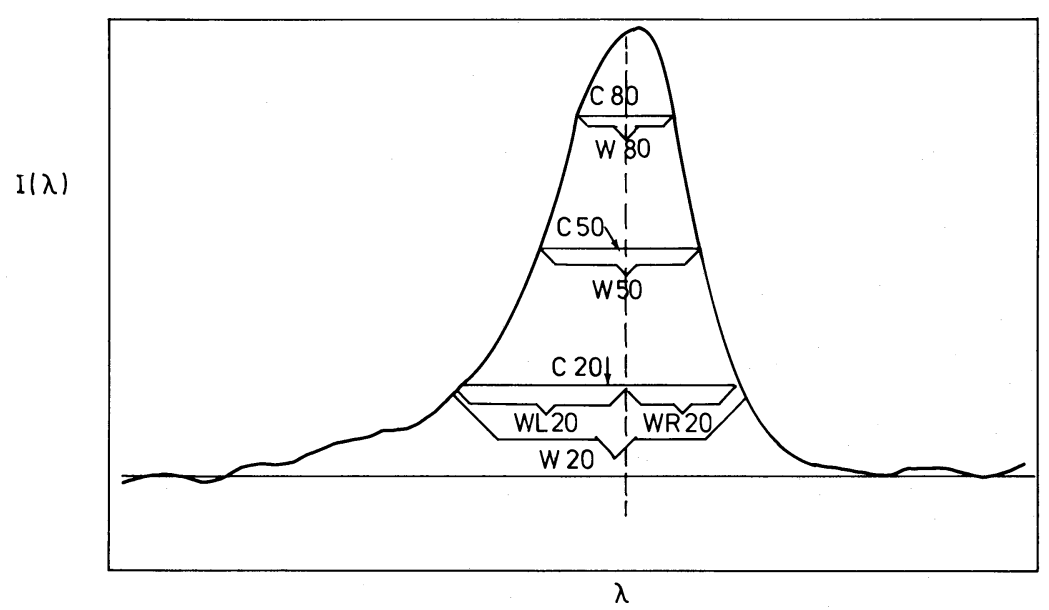

FIG. 2.- Schematic representation of the various line parameters defined in the text $\left(\mathrm{C} 80, \mathrm{C} 50, \mathrm{C} 20, \mathrm{~W} 80, \mathrm{~W} 50, \mathrm{~W} 20, \mathrm{AI}_{20}\right)$. The dashed vertical line indicates the position of $\mathrm{C} 80$, and the relative shifts of $\mathrm{C} 50$ and $\mathrm{C} 20$ are indicated by tick marks.

fully checked our data for such effects. First, the strongest nonsaturated lines in the He-Ne-Ar comparison spectra were examined and found to be highly symmetric. Second, we have plotted both $\mathrm{AI}_{20}$ and the difference between the [O III] velocity and the galaxy systemic velocity (see below) as a function of the [O III] line strength, since stronger lines would be expected to be most seriously affected. No correlation was found in either case.

Note that we have measured the [O III] $\lambda 5007$ profile of NGC 1068 published by Pelat and Alloin (1980) in the same manner as described above and have included it in our analyses below.

\section{GENERAL RESULTS}

\section{a) Line Asymmetries and Shifts}

Examination of Figure 1 and Table 1 clearly shows that the [O III] line is usually asymmetric in the sense of having a less steep falloff of intensity to the blue side of the peak. The effect can be most clearly seen in Mrk 279, NGC 1275, and Mrk 609 but is definitely present $(\geq 2 \sigma)$ in $\gtrsim 62 \%$ ( 23 out of 37 ) of the galaxies studied. In contrast only one object, Mrk 270 , definitely appears to exhibit an asymmetry in the opposite sense. A histogram of the asymmetry index $\mathrm{AI}_{20}$ (defined in $\S$ III) is shown in Figure 3. This asymmetry increases near the base of the profile (e.g., $\mathrm{AI}_{20}>\mathrm{AI}_{50}$ ) in $83 \%$ of the lines showing a blue asymmetry.

Although it is not obvious from Figure 1, inspection of expanded plots of the [O $\mathrm{III}] \lambda 5007$ profiles indicates that the profiles often have two component structures with a narrow "core" superposed on a broad, blueshifted "base."

The blueward asymmetries in the lines are accompanied by net blueshifts for the lines relative to the galaxy's systemic velocities in all cases where a comparison can

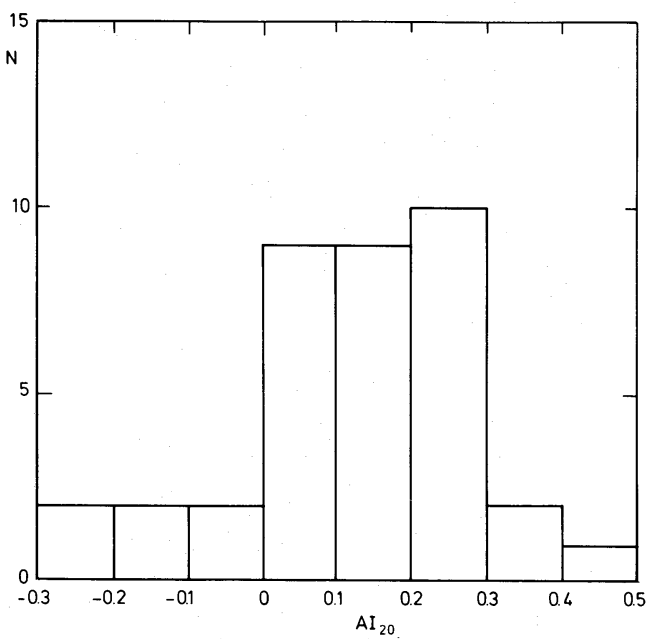

FIG. 3.- Histogram of the asymmetry index $\left(\mathrm{AI}_{20}\right)$ as defined in the text and Fig. 2 for galaxies in our sample.

be made. This is shown in Table 2. The sources for the line velocities and the systemic velocities (global $\mathrm{H}$ I profiles, stellar and interstellar absorption lines, and emission lines from extranuclear $\mathrm{H}$ II regions) are listed. We have not compared $V_{\text {SYS }}$ with our [O III] velocities in Table 1 for Mrk 10 and NGC 3227 ([O III] velocities are not accurately measured), NGC 7469 , Mrk 176, and Mrk 463 (interacting galaxies in which $V_{\mathrm{H}_{\mathrm{I}}}$ may not indicate $V_{\text {SYS }}$ ), and Mrk 3 (H I found by de Bruyn 1980 to be in a gas-rich companion galaxy).

It seems likely that the blueward asymmetries in the line profiles and their net blueshifts have a common origin. An obvious explanation is that the redward wings of the line profiles are being preferentially attenuated. This situation would naturally occur if the emitting gas is mixed with dust and flowing radially with respect to the nucleus. Such a picture has been sketched previously by Grandi (1977) on the basis of the blueward shifts and 
TABLE 2

COMParison OF NARRow LiNe aNd SySTEMIC Velocities

\begin{tabular}{|c|c|c|c|}
\hline Galaxy & $\begin{array}{c}\mathrm{C} 50-V_{\mathrm{SYS}} \\
\left(\mathrm{km} \mathrm{s}^{-1}\right)\end{array}$ & C50 References & $V_{\text {SYS }}$ References \\
\hline NGC 1068 . & -150 & Pelat and Alloin 1980 & Heckman et al. 1978 \\
\hline NGC $1275 \ldots$ & -280 & This paper & Rubin et al. 1977 \\
\hline NGC $3227 \ldots$ & -180 & Rubin and Ford 1968 & Rubin and Ford 1968 \\
\hline NGC $4051 \ldots$ & -105 & This paper & Heckman et al. 1978 \\
\hline NGC $4151 \ldots$ & -90 & This paper & Heckman et al. 1978 \\
\hline NGC 5548 . & -175 & This paper & Heckman et al. 1978 \\
\hline NGC 6764 & -50 & Rubin et al. 1975 & Heckman et al. 1978 \\
\hline IC $4329 \mathrm{~A}$ & -60 & This paper & Wilson and Penston 1979 \\
\hline Mrk $79 \ldots$ & -10 & This paper & Heckman et al. 1978 \\
\hline
\end{tabular}

asymmetries shown by the emission lines in PKS $1345+$ 12 and by Pelat, Alloin, and Fosbury (1980) for NGC 3783. Further evidence that dust is a significant constituent of the NLRs in Seyfert galaxies comes from measurements of optical polarization (e.g., Thompson et al. 1980; Schmidt and Miller 1980) and from studies of relative intensities of the narrow emission lines (e.g., Neugebauer et al. 1980).

To investigate this possibility, we compared our line asymmetries with the $\mathrm{H} \alpha / \mathrm{H} \beta$ narrow emission-line ratios. These were taken from the spectrophotometric references listed in $\S \mathrm{I}$ and are presumably a good measure of the amount of dust present in the NLR. The resultant plot shown in Figure 4 demonstrates the existence of a striking relationship and provides powerful evidence that dust is indeed a major influence in determining the shape of the emergent line profiles.

An intriguing relation also exists between the degree of line asymmetry and the ionization state for the class 1

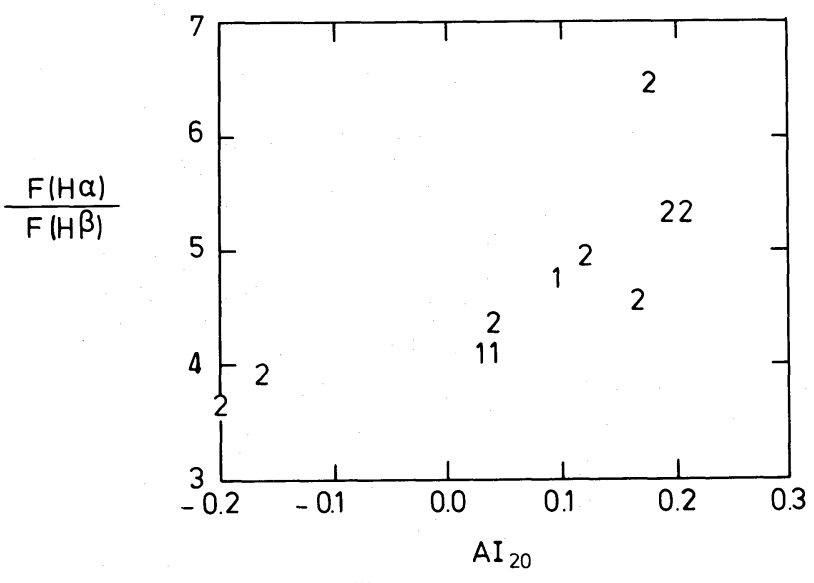

FIG. 4
Seyferts. Figure 5 shows a clear inverse correlation between $\mathrm{AI}_{20}$ and both the relative intensities of the $\left[\begin{array}{ll}\mathrm{O} & \mathrm{III}\end{array}\right] \lambda 5007 /\left[\begin{array}{ll}\mathrm{O} & \mathrm{II}\end{array}\right] \lambda 3727$ and [O $\left.\mathrm{OII}\right] \lambda 5007 /\left[\begin{array}{ll}\mathrm{O} & \mathrm{I}\end{array}\right]$ $\lambda 6300$ lines. Since reddening due to dust will affect the $\left[\begin{array}{ll}\mathrm{O} & \mathrm{II}\end{array}\right] /[\mathrm{O} \mathrm{II}]$ ratio in an opposite sense to the [O $\left.\mathrm{III}\right] /$ $[\mathrm{O} \mathrm{I}]$ ratio, the correlation seen above between $\mathrm{AI}_{20}$ and reddening should not systematically bias this figure. This relation holds only for objects which have broad permitted-line components (class 1 Seyferts and broadline radio galaxies). A similar plot for the class 2 Seyferts and narrow-line radio galaxies is a scatter diagram. The implications of this are discussed in $\S$ VI below.

The good correlations shown between $\mathrm{AI}_{20}$ and $\mathrm{H} \alpha / \mathrm{H} \beta$ (for all our galaxies) and between $\mathrm{AI}_{20}$ and the $\left[\begin{array}{ll}\mathrm{O} & \mathrm{III}\end{array}\right] /\left[\begin{array}{ll}\mathrm{O} & \mathrm{II}\end{array}\right]$ and $\left[\begin{array}{ll}\mathrm{O} & \mathrm{III}\end{array}\right] /\left[\begin{array}{ll}\mathrm{O} & \mathrm{I}\end{array}\right]$ flux ratios (for the galaxies having broad permitted lines) are further evidence that the line profiles are not seriously influenced by systematic measurement errors (e.g., beam-pulling, as discussed above).

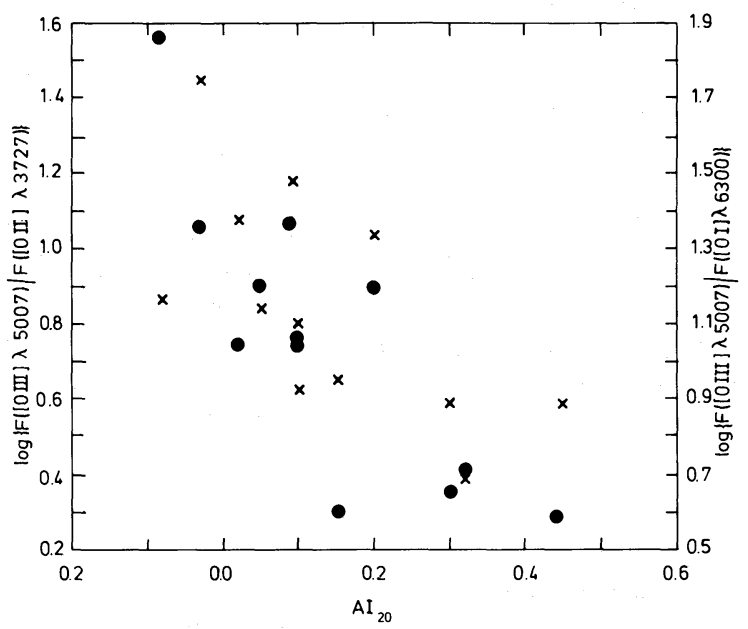

FIG. 5

FIG. 4. - The ratio of the fluxes of the narrow $\mathrm{H} \alpha$ and $\mathrm{H} \beta$ lines plotted against the asymmetry index $\left(\mathrm{AI}_{20}\right)$

FIG. 5.- The $\log$ of the flux ratio of the [O $\mathrm{III}] \lambda 5007$ to [O $\mathrm{II}] \lambda 3727$ lines (left-hand scale and dots) and the log of the flux ratio of the [O III] $\lambda 5007$ to $[\mathrm{O} \mathrm{I}] \lambda 6300$ lines (right-hand scale and crosses) plotted versus the asymmetry index $\left(\mathrm{AI}_{20}\right)$ for objects having a broad-line region. 
To investigate possible connections between the degree of asymmetry and the physical conditions of the line-emitting gas, we searched without success for correlations of $\mathrm{AI}_{20}$ with the characteristic electron temperature $T_{e}\{$ from $I([\mathrm{O} \mathrm{III}] \lambda 4363) / I([\mathrm{O} \mathrm{III}] \lambda 4959+5007)\}$ or with the electron density $N_{e}$ (from $I([\mathrm{~S}$ II $] \lambda 6717)$ ) $\left.I\left(\left[\begin{array}{l}\mathrm{S} \\ \mathrm{II}\end{array}\right] \lambda 6731\right)\right\}$. For this purpose, line ratio data were taken from Osterbrock, Koski, and Phillips (1976); Costero and Osterbrock (1977); Osterbrock (1977); Koski (1978).

Although (as we shall see below) there is a difference in the characteristic line widths between galaxies that have a broad permitted-line component and those that do not, we find no systematic difference in the asymmetry indices between the two classes.

One parameter that might be influenced by dust in the nucleus is the infrared luminosity. Comparing the nuclear infrared luminosities of Rieke (1978) with our line asymmetries and widths, we find no evidence of any relationship. However, this is not too surprising since the infrared luminosity would be influenced by the presence of a nonthermal continuum source and by the bolometric luminosity of the nucleus as well as by the dust. If the infrared luminosity is taken as representative of the nuclear bolometric luminosity (cf. Rieke 1978), it is then interesting that more luminous objects do not exhibit broader [O III] lines. The implications of this will be discussed below.

We also fail to find any correlation between line asymmetries and line widths, implying that the amount of dust in the NLR is not closely related to the kinematics.

As mentioned above, the optical polarization seen in Seyfert nuclei is generally attributed to scattering by nuclear dust particles. We have therefore searched for a correlation between $\mathrm{AI}_{20}$ and the fractional broad-band polarization measured by Maza (1979) for the 25 galaxies common to both samples. We find no significant correlation-which is not entirely unexpected, since the degree of polarization may depend more strongly on departures by the gas and dust from spherical symmetry than on the amount of dust present. Moreover, the dust responsible for absorbing the light may be distinct from the dust responsible for scattering (polarizing) the light.

Finally, we searched without success for a correlation between the line asymmetries and the inclinations of the surrounding galaxies for those Seyferts whose orientation has been measured (Keel 1980).

\section{b) Line Widths}

The distribution of $W_{50}$ is shown in Figure 6. This clearly demonstrates that galaxies that have broad components in their permitted lines (class 1 Seyferts and broad-line radio galaxies) have on average narrower [O III] lines than those that do not (class 2 Seyferts and narrow-line radio galaxies). The means of the two distri-

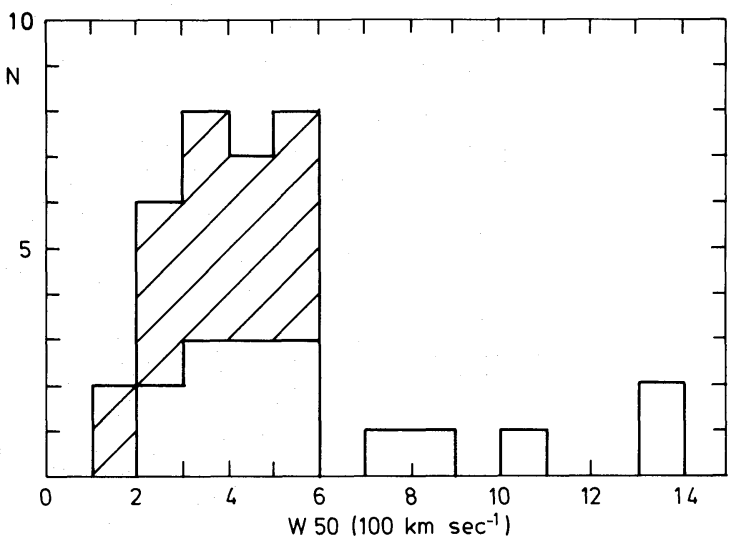

FIG. 6.- Histogram of the line widths defined at half the peak intensity $\left(W_{50}\right)$ in units of $100 \mathrm{~km} \mathrm{~s}^{-1}$. The objects that do not have broad permitted lines are plotted without hatching, while those that do are plotted with hatching.

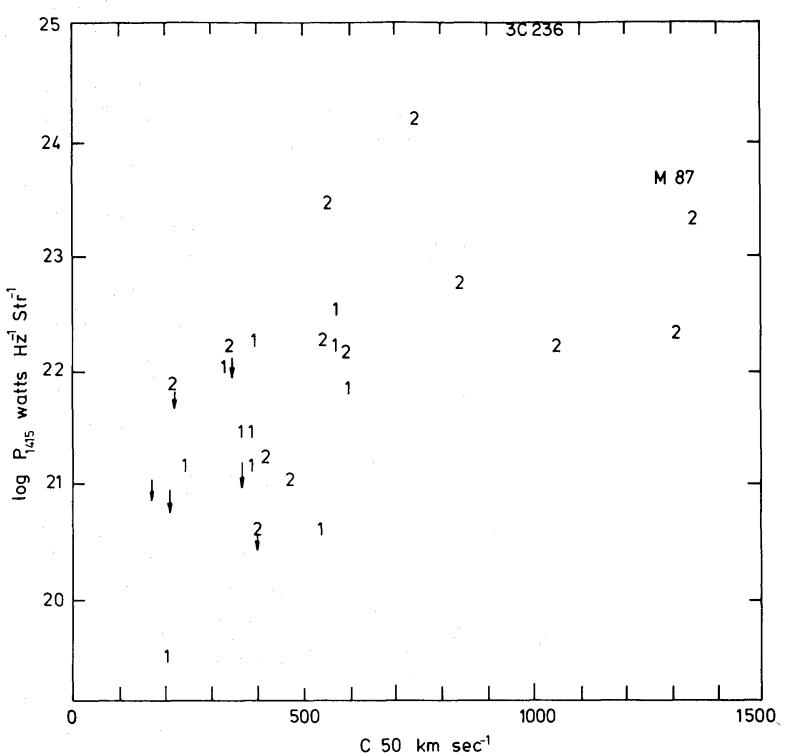

FIG. 7.-Logarithm of the monochromatic radio power at 1415 $\mathrm{MHz}$ (in watts $\mathrm{Hz}^{-1} \mathrm{sr}^{-1}$ ) of the kiloparsec-sized, steep-spectrum core sources plotted against the width of the [O III] $\lambda 5007$ line at half-maximum intensity (W50).

butions are $380 \pm 30$ and $620 \pm 90 \mathrm{~km} \mathrm{~s}^{-1}$, respectively. The latter value agrees well with that of Koski (1978) from less precise measurements of galaxies of this type.

We have also used our line width data to investigate the correlation between line width and radio power for Seyfert 2 galaxies as found by Wilson and Willis (1980) on the basis of Koski's line widths. Our more accurate data confirm this relation and extend it to the steepspectrum, kiloparsec-sized, radio cores of Seyfert 1 and radio galaxies. In Figure 7 we have plotted the line widths against the radio luminosities for galaxies in Table 1 for which appropriate radio data are available 
(Kellermann, Pauliny-Toth, and Williams 1969; Graham 1970; Miley and Perola 1975; de Bruyn and Wilson 1976; Fomalont, Miley, and Bridle 1979). In addition we have included the radio galaxies M87 (Virgo A) and 3C 236 using the line widths determined by Heckman, Balick, and Crane (1980) and by Miley and Osterbrock (1979), respectively. We emphasize that in every case only the radio power of the steep-spectrum, kiloparsecsized component (e.g., Miley 1980) has been used. We have excluded both the contribution of the extended lobes in the radio galaxies (typical sizes $\gtrsim 100 \mathrm{kpc}$ ) as well as the ultracompact "flat spectrum" cores (typical sizes $\lesssim 1 \mathrm{pc}$ ).

Figure 7 shows no strong indication that at a given radio luminosity the class 1 Seyferts have systematically narrower lines. Thus the difference between line widths in the class 1 and class 2 Seyferts shown in Figure 6 and the lower radio power of most class 1 Seyferts (cf. de Bruyn and Wilson 1976, 1978) are probably not independent phenomena.

We find no relationship between the line width and the inclination of the galaxy (cf. Keel 1980), indicating that the gas flow is not generally taking place in a preferred direction related to the large-scale properties of the galaxy.

There have been several reports of differences between the widths and/or relative velocities of lines produced by different ionic species in Seyfert galaxies, generally in the sense that species having larger ionization potentials have broader and/or more blueshifted line profiles (Phillips 1976; Koski 1978; Grandi 1978; Davidson and Kinman 1978; Wilson 1979; Pelat, Alloin, and Fosbury 1980).

We have therefore searched our data for differences between the [O III] $\lambda 5007$ profile and the narrow component of the $\mathrm{H} \beta$ profile. This comparison is plagued by two difficulties that will make our conclusions tentative. First, our $\mathrm{H} \beta$ data are often of low signal-to-noise ratio. Second, accurate deblending of the broad and narrow $\mathrm{H} \beta$ components is often difficult for class 1 Seyferts. Comparisons of line width and velocity for those cases in which the data warrant it, are summarized in Table 3. The objects are grouped according to whether the $\mathrm{H} \beta$ profile is wider, has approximately the same width, or is narrower than the corresponding [O III] $\lambda 5007$ profile. In the cases of NGC 1275 and Mrk 609 (discussed in detail below) the differences in the widths are very striking, while in the other cases the differences are typically only $15 \%$. It would appear from Table 3 that when the $\mathrm{H} \beta$ and [O III] profiles differ in width, the wider line is significantly blueshifted ( $\gtrsim 30 \mathrm{~km} \mathrm{~s}^{-1}$ ) compared with the narrower line. A similar result has been reported for NGC 3783 by Pelat, Alloin, and Fosbury (1980). Higher quality observations are required to confirm this effect, and we will therefore refrain from further discussion of its significance.
TABLE 3

Comparison of THE [O III $] \lambda 5007$ and H $\beta$ PROFILes

\begin{tabular}{|c|c|c|}
\hline Galaxy & $\begin{array}{c}V_{\mathrm{H} \beta}-V_{\left[\mathrm{O}_{\mathrm{III}}\right]} \\
\left(\mathrm{km} \mathrm{s}^{-1}\right)\end{array}$ & $\begin{array}{l}\mathrm{H} \beta \text { versus } \\
{[\mathrm{O} \mathrm{III}] \text { Width }}\end{array}$ \\
\hline Mrk $6 \ldots$ & -83 & Wider \\
\hline Mrk 78 . & -69 & Wider \\
\hline Mrk $298 \ldots \ldots$ & -7 & Wider \\
\hline Mrk 3 & 15 & Same \\
\hline Mrk 34 & -10 & Same \\
\hline Mrk $40 \ldots$ & -47 & Same \\
\hline Mrk $766 \ldots \ldots$ & 6 & Same \\
\hline $3 \mathrm{C} 390.3 \ldots \ldots$ & -14 & Same \\
\hline NGC $1275 \ldots$ & 280 & Narrower \\
\hline NGC $2992 \ldots$ & 4 & Narrower \\
\hline NGC 4151 . & 40 & Narrower \\
\hline NGC 7469 & 104 & Narrower \\
\hline Mrk $270 \ldots$ & -2 & Narrower \\
\hline Mrk 463. & 63 & Narrower \\
\hline Mrk 609 . & 130 & Narrower \\
\hline 3C $98 \ldots \ldots \ldots$ & 94 & Narrower \\
\hline Cyg A ........ & 28 & Narrower \\
\hline
\end{tabular}

\section{INDIVIDUAL INTERESTING OBJECTS}

a) NGC 1275

It is evident from Figure 1 that in NGC 1275 the [O III] and $\mathrm{H} \beta$ lines have different profiles. Both lines appear to consist of two components: a narrow one $\left(\mathrm{FWHM} \approx 400 \mathrm{~km} \mathrm{~s}^{-1}\right)$ at $V \approx 5200 \mathrm{~km} \mathrm{~s}^{-1}$, and a broad one $\left(F W H M \approx 2000 \mathrm{~km} \mathrm{~s}^{-1}\right)$ which is blueshifted by some $300-400 \mathrm{~km} \mathrm{~s}^{-1}$.

The narrower component is relatively more prominent in $\mathrm{H} \beta$ than in [O III], and this is the principal difference between the two profiles. Balick and Heckman (1979) have previously noted that the [O II] $\lambda 3727$ line is much narrower than the [O $\mathrm{III}] \lambda 5007$ line in NGC 1275 and have suggested that the [O II] line comes predominantly from the well-known, extended filamentary emission-line system, while the [O III] line is produced in the semistellar Seyfert nucleus. Similarly, the difference between the [O III] and $\mathrm{H} \beta$ profiles can be understood if the narrow components of the profiles arise in the extended nebulosity, which has a low excitation state ([O III] $\lambda 5007 / \mathrm{H} \beta$ intensity ratio $\sim 0.6$ according to Kent and Sargent 1979). The broad component would then arise in the high excitation gas in the nucleus.

\section{b) Markarian 298=IC 1182}

Mrk 298 was observed during good seeing, and here the difference between the $\mathrm{H} \beta$ and [O III] profiles is definitely due to the superposition of several components that have different relative strengths of [O III] to $\mathrm{H} \beta$ and that are kinematically and spatially distinct. The two-dimensional spectrum is shown in Figure 8, 


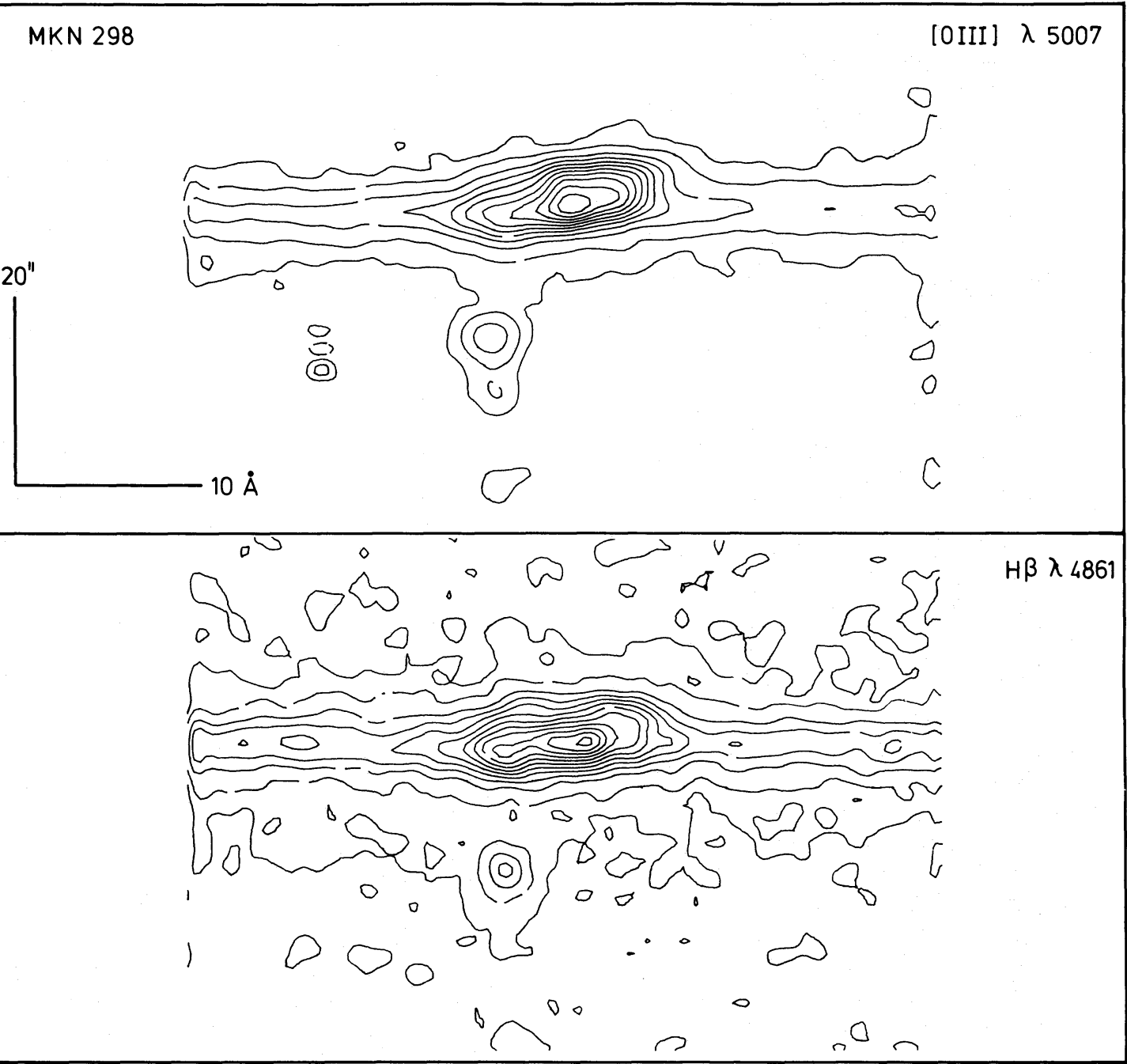

FIG. 8. - Contour plots of the distributions of the [O III] $\lambda 5007$ and $\mathrm{H} \beta \lambda 4861$ lines in Mrk 298. The slit was oriented in a position angle of $100^{\circ}$ with east at the bottom of the figure. The contours are in (arbitrary) counts pixel ${ }^{-1} \mathrm{~s}^{-1}$ at levels of 20,60 , and $60+80 \mathrm{~N}$ above the sky in the [O III] plot, and 20,30, and 30+40 N above the sky in the $\mathrm{H} \beta$ plot. The scale in arcseconds and angstroms is illustrated.

where the nuclear emission-line region is seen to break up into a multicomponent complex, distributed over $\sim 5^{\prime \prime} 5(\sim 3.8 \mathrm{kpc})$, along a position angle of $100^{\circ}$. These components span a velocity range of $\sim 350 \mathrm{~km} \mathrm{~s}^{-1}$. Faint emission from the knotty, jetlike protrusion to the east of Mrk 298, which has previously been noted by Stockton (1968), can also be seen.

Figure 8 shows only the innermost part of this protrusion. The lines from individual knots in this feature- the westernmost of which can be seen $\sim 13^{\prime \prime}$ below the nucleus in Figure 8-are unresolved (FWHM $\lesssim 100 \mathrm{~km}$ $\mathrm{s}^{-1}$ ). Proceeding eastward (and thus below the portion of the spectrum shown in Fig. 8), the feature shows a fairly steady redward velocity gradient amounting to $\sim 100 \mathrm{~km} \mathrm{~s}^{-1}$ over $75^{\prime \prime}(\sim 50 \mathrm{kpc})$. As can be seen in Figure 8, the knot nearest the nucleus in the protrusion has the same velocity as the easternmost part of the nuclear cloud complex and is apparently connected to it by faint emission at the same velocity. There seems then to be little doubt that the spectroscopic complexity of the nucleus is related in some way to the presence of the jetlike feature.

Adams (1977) and Wehinger and Wyckoff (1977) have found a surprisingly large fraction of Seyfert galaxies to show signs of gravitational interaction. Since Mrk 298 is located in a region of high galaxy density (the Hercules cluster), it is conceivable that the protrusion and some of the nuclear clouds are tidal debris of a late-type galaxy which is interacting with Mrk 298. However, the relatively quiescent kinematics exhibited by the protrusion may not be consistent with this explanation. 


\section{c) Markarian 609}

This is a remarkable object. The $\mathrm{H} \beta$ profile is only $\sim 110 \mathrm{~km} \mathrm{~s}^{-1}$ wide (barely resolved), while the [O III] lines are about 4 times wider $\left(W_{50}=450 \mathrm{~km} \mathrm{~s}^{-1}\right)$. Moreover, the broad, blueshifted wings seen on the [O III] lines are completely missing in $\mathrm{H} \beta$. Clearly, the $\mathrm{H} \beta$ and [O $\mathrm{III}]$ lines are coming from two quite distinct regions: one in which the velocities are large and $I([\mathrm{O} \mathrm{III}]) \gg I(\mathrm{H} \beta)$, and one in which the kinematics are much more quiescent and $I([\mathrm{O} \mathrm{III}]) \ll I(\mathrm{H} \beta)$. It is possible that the [O III] lines are representative of the Seyfert nucleus, and that $\mathrm{H} \beta$ comes from extremely luminous giant $\mathrm{H}$ II regions near the nucleus. The near-nucleus H II regions would be expected to have $I(\mathrm{H} \beta) \gg I$ ([O III]) according to current ideas (e.g., Heckman $1980 a$ ). Suggestively, the $\mathrm{H} \beta$ lines are redshifted relative to the [O III] lines by $\sim 130 \mathrm{~km} \mathrm{~s}^{-1}$, as would be expected from our earlier discussion if they arose in $\mathrm{H}$ II regions with $V \approx V_{\text {SYS }}$.

\section{d) Markarian 42}

Markarian 42 is a particularly fascinating object. It was originally listed as a class 2 Seyfert on the basis of the narrowness of the permitted lines. However, Koski (1978) showed it to have a spectrum that strongly resembled that of a class 1 Seyfert (e.g., the spectrum was dominated by permitted lines, including strong $\mathrm{Fe}$ II emission, and had a strong "nonthermal" continuum component). He suggested that it was, in fact, a class 1 Seyfert with unusually narrow lines from its "broad-line" region. Our spectra confirm this idea. The $\mathrm{H} \beta$ profile clearly shows a weak, base component that is missing in the [O III] $\lambda 5007$ profile. This base is exceptionally narrow for a class 1 Seyfert, having a full width at zero intensity of only $\sim 4000 \mathrm{~km} \mathrm{~s}^{-1}$ (cf. Osterbrock 1977). Even the very narrow core of the $\mathrm{H} \beta$ line is far stronger relative to [O III] $\lambda 5007$ than the $10 \%$ value typically found in the narrow-line region of Seyferts (Koski 1978). Perhaps then much of this narrow core is also produced in the broad-line region in Mrk 42.

One explanation for the spectrum of Mrk 42 (narrow lines coupled with Seyfert 1 line ratios) is that the lines appear narrow due to a projection effect (e.g., a BLR in the form of a rotating disk seen nearly pole-on as discussed by Osterbrock 1977).

\section{e) Markarian 78}

The lines are clearly double-peaked (peak separation $\sim 730 \mathrm{~km} \mathrm{~s}^{-1}$ ). Spectra and photographs by Adams (1973) have shown the core of the NLR to consist of two distinct clouds separated by $\sim 2^{\prime \prime}(\sim 1.5 \mathrm{kpc})$ in position angle $\sim 100^{\circ}$. Since our slit was set at position angle $=0^{\circ}$ for these observations, no information on the relative kinematics of the clouds was obtained. However, it is tempting to identify the two clouds with the two peaks in the line profile. These clouds seem to be associated with a double nuclear radio source that has the same separation size and position angle (Wilson and Willis 1980).

\section{f) $3 C 305$}

This radio galaxy also shows a double-peaked [O III] line with the peaks separated by $\sim 460 \mathrm{~km} \mathrm{~s}^{-1}$. As in Mrk 78, the gas seems intimately related to a nuclear radio source, and here the double-peaked profile is definitely a direct result of the large-scale kinematics of the NLR. We have carried out a detailed optical and radio investigation of this galaxy which will be described in a forthcoming paper.

\section{g) NGC 2992}

This galaxy, which is in an interacting system with NGC 2993, has been discussed by Burbidge et al. (1972), Osmer, Smith, and Weedman (1974), and Ward et al. (1978). NGC 2992 is particularly interesting from the standpoint of investigations of the NLR because it is seen nearly edge-on (as judged from the dust band that bisects the galaxy), so that information concerning the distribution and kinematics of the NLR gas located out of the galaxy plane can be inferred. Moreover, unlike the other edge-on Seyferts (IC 4329A and NGC 4235), the NLR in NGC 2992 is well resolved spatially (Heckman and Balick 1981), thus facilitating such investigations.

Our spectrum (obtained at position angle $120^{\circ}$, or roughly perpendicular to the dust lane) shows [O III] emission extending over $\sim 30^{\prime \prime}$ (or $\sim 4.5 \mathrm{kpc}$ for a Hubble constant $H_{0}=75$ ). Both the continuum and [O III] intensities are larger to the east of the dust band, and thus we identify this as the near side of the galaxy (a choice which is also supported by the unpublished images of NGC 2992 obtained by Heckman and Balick).

Figure 9 is a representation of the NGC 2992 spectrum, while Figure 10 shows the run of velocity and line width along the slit derived from this spectrum. Although the kinematics are evidently quite complex, several simple points can be made.

First, Figure 10 shows that far $\left(10^{\prime \prime}-14^{\prime \prime}\right)$ from the nucleus the velocities to the east-southeast (near side) are $\sim 140 \mathrm{~km} \mathrm{~s}^{-1}$ less than at a similar distance to the west-northwest (far side). Thus, if radial flow is responsible for this difference, the flow must be outward.

Second, Figure 10 demonstrates that the gas velocities within $\sim 4^{\prime \prime}$ from the nucleus are blueshifted by $\gtrsim 100$ $\mathrm{km} \mathrm{s}^{-1}$ compared with the mean of the velocities far from the nucleus. Since we saw earlier (Table 2) that the [O III] nuclear velocity is typically blueshifted by $\sim 100$ $\mathrm{km} \mathrm{s}^{-1}$ relative to the systemic velocity of the galaxy for Seyferts, this result for NGC 2992 can be easily understood if the mean gas velocity far from the nucleus is close to the systemic velocity of the galaxy. 


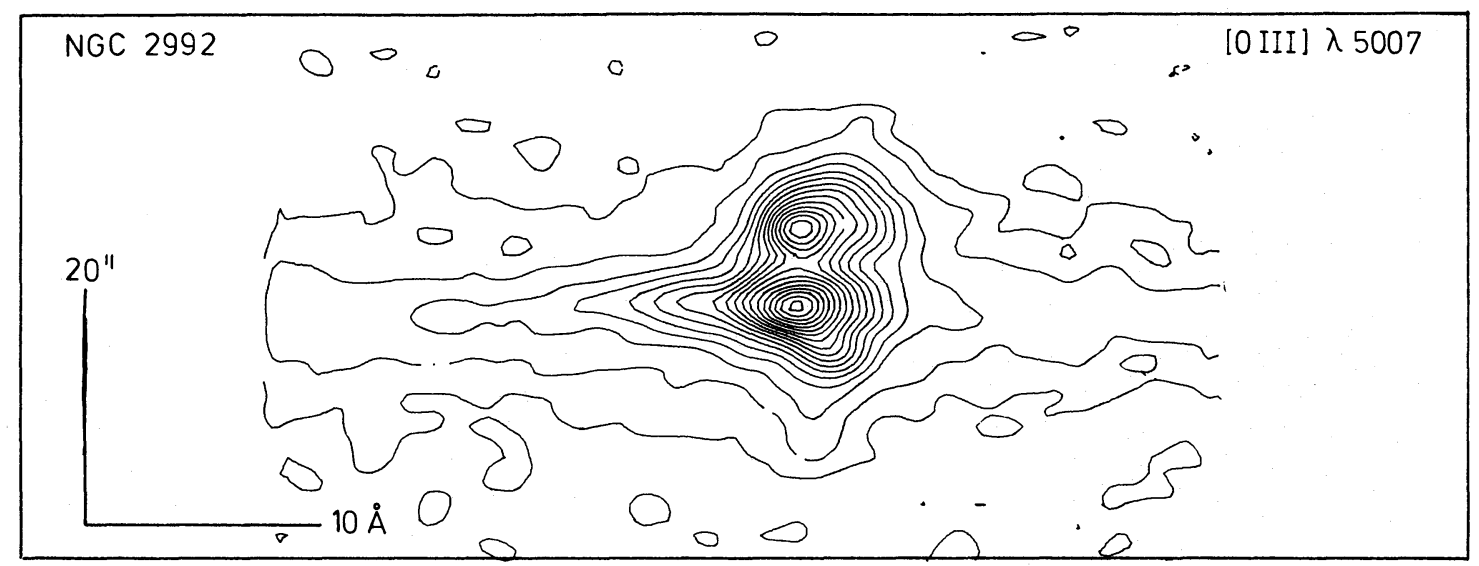

FIG. 9. - Contour plot of the distribution of the [O $\mathrm{III}] \lambda 5007$ line in NGC 2992. The slit was oriented in a position angle of $120^{\circ}$, and east is to the bottom of the figure. The contours are in counts $\mathrm{s}^{-1} \mathrm{pixel}^{-1}$ at levels of 40,120 , and $120+160 \mathrm{~N}$ above the sky. The scale in arcseconds and angstroms is illustrated.

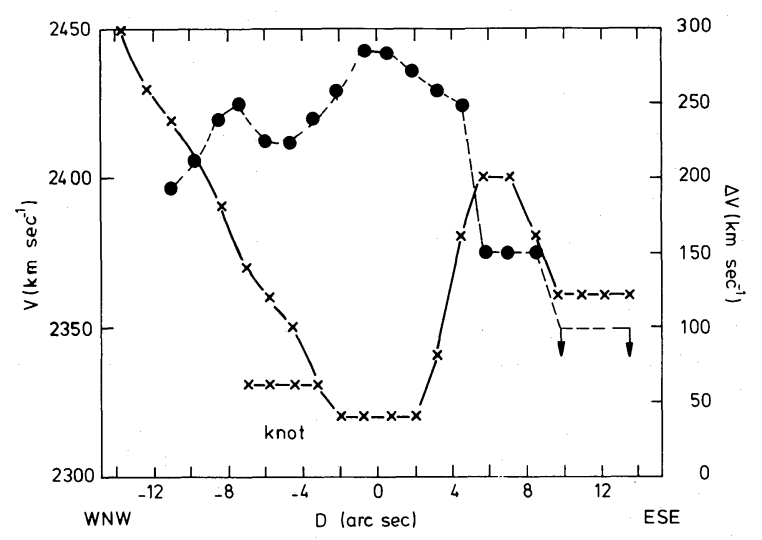

FIG. 10. - Plot of the velocity (solid line, crosses, and left-hand scale) and the line width (dashed line, dots, and right-hand scale) of the $[\mathrm{O} \mathrm{III}] \lambda 5007$ line as a function of the projected distance from the nucleus for NGC 2992. The velocity of a bright knot located $\sim 5^{\prime \prime}$ to the west-northwest of the nucleus is also shown.

Third, it is clear from Figure 10 that the [O III] line in NGC 2992 remains wide $\left(\Delta V>100 \mathrm{~km} \mathrm{~s}^{-1}\right)$ far from the nucleus. This is especially true to the west-northwest (far side). Either the extended gas is relatively turbulent in NGC 2992 or the apparent width of the line arises geometrically (e.g., outflow into a wide, conelike pattern with a single line-of-sight intersecting gas in which only the projected velocity component in the line of sight varies).

Fourth, a conspicuous, broad "blue wing" on the [O III] profile can be seen (Fig. 9) confined to the innermost few arcseconds of the NLR. We identify this with the broad, blueshifted "base" component of the line profiles discussed above.

The complexity of the kinematics shown by the gas precludes any more detailed discussion based on our limited data, but suggests that a thorough investigation of the NLR in NGC 2992 would be very instructive.

\section{h) NGC 4151}

This nearby, bright galaxy is (along with NGC 1068) the most thoroughly studied Seyfert. Several previous investigations of the NLR kinematics have been conducted (e.g., Ulrich 1973; Fricke and Reinhardt 1974). This previous work has suggested that the inner $\sim 300$ pc of the NLR consists of several spatially/kinematically distinct clouds, and that noncircular motions are present even well beyond this distance.

Our spectrum at position angle $45^{\circ}$ (the most dynamically interesting one according to the above references) is shown in Figure 11, while Figure 12 displays the variation in velocity and line width along the slit. The situation here bears some similarity to NGC 2992 in that the velocities near the nucleus (within $\sim 4^{\prime \prime}$ ) are negative (by $\sim 100 \mathrm{~km} \mathrm{~s}^{-1}$ ) as compared with the mean of the velocities further out. The rotation axis of NGC 4151 is now known to be at position angle $\sim 120^{\circ}$ (Bosma, Ekers, and Lequeux 1977), so that the offset of $120 \mathrm{~km} \mathrm{~s}^{-1}$ between the gas to the northeast and southwest, seen in Figure 11, can be understood in terms of simple rotation. The blueshift of the gas at $r \leq 4$ ".5 could then be explained by the "radial flow plus dust" picture described earlier.

Bosma, Ekers, and Lequeux (1977) measure a constant deprojected rotation velocity of $\sim 150 \mathrm{~km} \mathrm{~s}^{-1}$ far out in the disk of NGC 4151. This agrees quite well with the value of $\sim 170 \mathrm{~km} \mathrm{~s}^{-1}$, which we would derive by assuming that the optically emitting gas at $4^{\prime \prime} .5 \leq r \leq 17^{\prime \prime}$ is in rotation and by using the same values for inclination $\left(21^{\circ}\right)$ and position angle of the dynamical axis $\left(\sim 120^{\circ}\right)$ as Bosma, Ekers, and Lequeux. This agreement, together with the narrow-line widths and regular velocities exhibited by the gas at $r>4.5$, strengthens the case for rotation.

However, we emphasize that the identification of the velocities of the optically emitting gas as being due to 


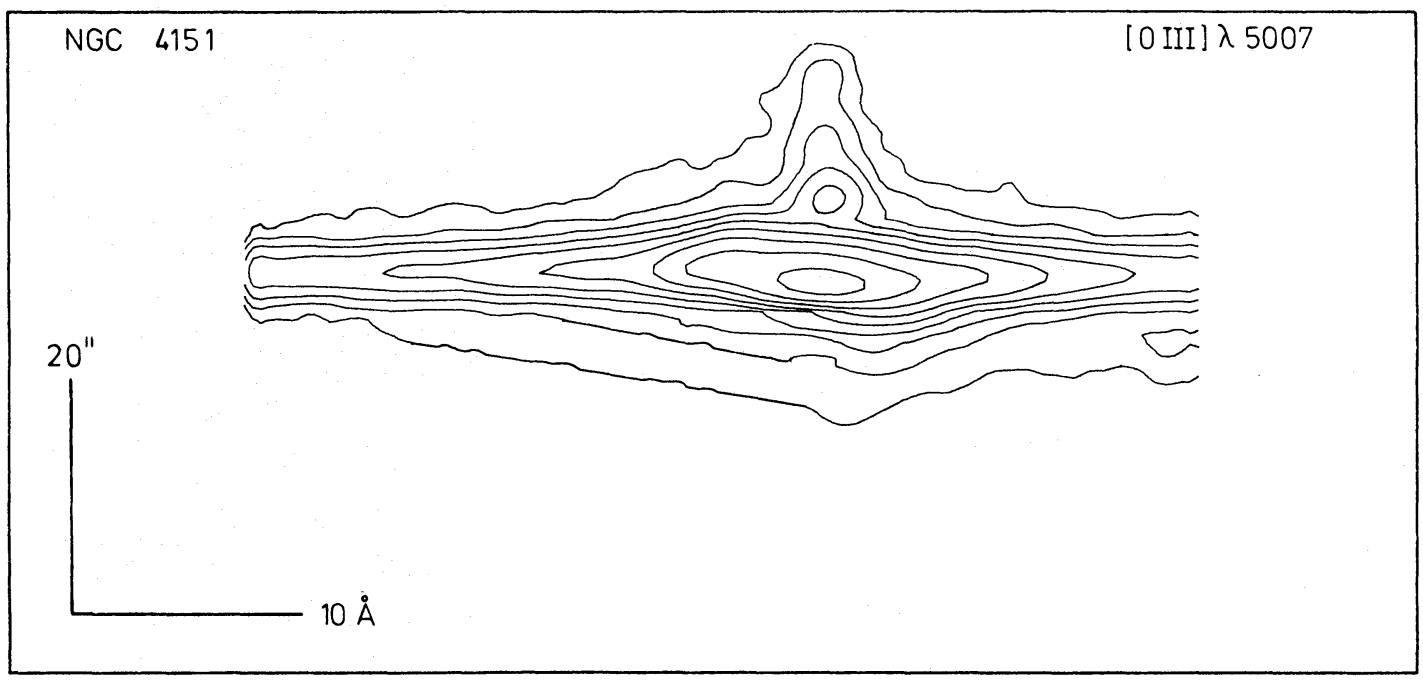

FIG. 11.-Contour plot of the distribution of the [O $\mathrm{III}] \lambda 5007$ line in NGC 4151 . The slit was oriented in a position angle of $45^{\circ}$, and northeast is to the bottom of the figure. The contours are in (arbitrary) counts $\mathrm{s}^{-1}$ pixel $^{-1}$ at logarithmically spaced levels of $60,120,240$, etc., above the sky. The scale in arcseconds and angstroms is illustrated.

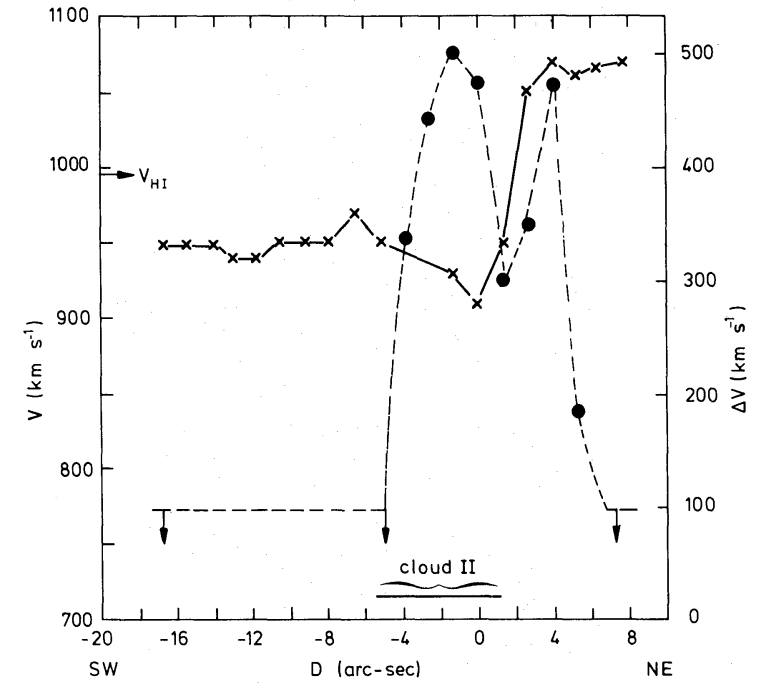

FIG. 12.-Plot of the velocity (solid line, crosses, and left-hand scale) and the line width (dashed line, dots, and right-hand scale) of the [O III] $\lambda 5007$ line as a function of projected distance from the nucleus of NGC 4151. The mean velocity of the $\mathrm{H}$ I profile as given by Heckman, Balick, and Sullivan (1978) is indicated, as is the location of Ulrich's (1973) Cloud II.

pure rotation is questionable until a more complete study is conducted. Although the data of Fricke and Reinhardt (1974) strongly suggest that noncircular velocities of up to $400 \mathrm{~km} \mathrm{~s}^{-1}$ are present out to a distance of $\sim 3 \mathrm{kpc}$ from the nucleus, their velocities are in very poor agreement with ours, and large errors are possible.

The kinematic structure of the inner $3^{\prime \prime}(200 \mathrm{pc})$ of the NLR is also interesting. First, careful inspection of
Figure 11 shows that the centroid of that portion of the NLR having the highest surface brightness is displaced by $\sim 0.7$ to the northeast (below, in Fig. 11) of the peak in the continuum brightness. However, the line profile at the position of the peak in the continuum brightness displays the characteristic blue wing most strongly, as can be seen in Figure 11. It is this position which serves as the reference in Figure 12.

Comparison of our data with that of Ulrich (1973) shows satisfactory agreement. Our derived velocity for the main core feature (her Cloud I) is $\sim 50 \mathrm{~km} \mathrm{~s}^{-1}$ smaller than hers. This is a reasonable discrepancy in view of the uncertainty of $\sim 30 \mathrm{~km} \mathrm{~s}^{-1}$ in each of the velocities.

We confirm the existence of Ulrich's Cloud II, which can be seen in Figure 11 as a "bulge" in the contours above and to the left of the nucleus. We find Cloud II to be blueshifted by $\gtrsim 200 \mathrm{~km} \mathrm{~s}^{-1}$ with respect to C50, and hence by $\gtrsim 275 \mathrm{~km} \mathrm{~s}^{-1}$ when compared with $V_{\mathrm{SYs}}$. (We give lower limits to the velocity because blending with the Cloud I profile probably leads to an underestimate of the true blueshift of Cloud II.) Examination of the [O III] profile in individual rows of the spectrum shown in Figure 11 shows that Cloud II begins near the nucleus and peaks in intensity about $1^{\prime \prime}(\sim 70 \mathrm{pc})$ to the southwest (above the nucleus in Fig. 11). Its total spatial extent is difficult to judge, but it can be traced from at least $5^{\prime \prime}$ southwest to $1 . .5$ northeast of the nucleus (and so is considerably larger than what is evident in Ulrich's photographic spectra). The line is probably spectroscopically resolved with a FWHM $\approx 150 \mathrm{~km} \mathrm{~s}^{-1}$, although, since it is badly blended with the Cloud I line profile, this estimate is rather uncertain. Whether the anomalous velocity of the cloud is due to inflow or 
outflow cannot be ascertained without knowledge of its three-dimensional location with respect to the nucleus.

\section{DISCUSSION}

\section{a) Kinematics of the NLR}

The striking asymmetries generally present in our [O III] line profiles provide direct information concerning the kinematics of the narrow-line region. The most obvious explanation for these asymmetries is that the line emission is produced by gas flowing outward at velocities of a few hundred kilometers per second, and that this gas is mixed with dust. The receding gas (on the far side) will then be preferentially hidden from the observer by the intervening dust, and a blue asymmetry in the line profile will result. Taking the velocities, masses, and scales characteristic of the NLRs, a mass flow rate of $\sim 1 M_{\odot} \mathrm{yr}^{-1}$ is typically involved.

However, it is possible to construct a picture in which dust plus infall can also produce the observed asymmetries in the line profiles. If the NLR consists of small, dusty, infalling clouds embedded in a relatively transparent medium, if, because they are so dusty, each cloud is ionized only on the side facing the continuum source (the nucleus), and if the "covering factor" in the NLR is less than 1 (so that clouds on the far side of the nucleus are not obscured from the observer by the clouds on the near side), then a blue asymmetry in the lines would result. The idea is just the converse of those recent models seeking to explain the red asymmetries in the BLR profiles in an outflow model (Ferland, Netzer, and Shields 1979; Capriotti, Foltz, and Byard 1979). Note that in these BLR models the asymmetry is produced by the absorption by neutral gas of the emission-line photons as they propagate through the part of the cloud facing away from the nucleus. In the case of the NLR forbidden lines, dust must be invoked, since the forbidden lines cannot be appreciably absorbed by gas alone. An optical depth of $\tau_{d} \approx 2$ at $\lambda 5000$, due to dust in each cloud, is typically implied by $\mathrm{H} \alpha / \mathrm{H} \beta$ (e.g., Koski 1978). This would then give $\tau_{d} \gtrsim 10$ for Lyman continuum photons, so that only the side of the cloud facing the nucleus would be ionized. Although this picture is somewhat contrived, infall, as opposed to outflow, cannot be excluded from only the blue asymmetry in the profiles.

However, one line of argument which favors outflow over infall in NLR is that the three $\mathrm{H} \mathrm{I}$ absorption lines detected from Seyfert galaxies (Heckman, Balick, and Sullivan 1978) all show blueshifts of several hundred kilometers per second compared with the systemic velocity of the galaxy (Mrk 6, $V_{\mathrm{ABS}}-\mathrm{C} 80=-140 \mathrm{~km} \mathrm{~s}^{-1}$, implying that $V_{\mathrm{ABS}}-V_{\mathrm{SYS}}<-140 \mathrm{~km} \mathrm{~s}^{-1}$; Mrk 231, $V_{\mathrm{ABS}}-V_{\mathrm{SYS}}=-270 \mathrm{~km} \mathrm{~s}^{-1}$ for $V_{\mathrm{SYS}}$ from Boksenberg et al. (1977); NGC 5548, $V_{\mathrm{ABS}}-V_{\mathrm{SYS}}=-380 \mathrm{~km} \mathrm{~s}^{-1}$ ). Since these velocities seem representative of the characteristic velocities of the NLR, it is reasonable to identify the absorbing material with neutral gas flowing out, along with the ionized gas of the NLR.

Furthermore, Bell and Seaquist (1980) have reported the detection of $\mathrm{H} 83 \alpha$ and $\mathrm{H} 99 \alpha$ radio recombination lines from the broad-line radio galaxy OQ 208 (=Mrk 668). These lines have a blueshift of $\sim 120 \mathrm{~km} \mathrm{~s}^{-1}$ compared with the velocity of the [O III] $\lambda 5007$ line (cf. Table 1). Several of Bell and Seaquist's conclusions suggest that the gas giving rise to the recombination lines is located in the NLR. The gas must be at least $150 \mathrm{pc}$ from the compact nuclear radio source, have an electron density $\sim 10^{4} \mathrm{~cm}^{-3}$, and have a filling factor less than $4 \times 10^{-4}$. These parameters are strikingly similar to those of the NLR. Since the emission mechanism suggested to explain the radio lines is emission stimulated by the compact nonthermal radio source in the nucleus, the emitting gas must be located in front of the nucleus. Thus these observations provide independent evidence for outflow at $V \gtrsim 10^{2} \mathrm{~km} \mathrm{~s}^{-1}$ in the NLR.

Another argument against infall is that the infalling material would almost certainly have much higher angular momentum than the nuclear gas. This excess angular momentum could be dissipated only very slowly in an axisymmetric disk system, so that infall at velocities of several hundred kilometers per second would be impossible. Such velocities of infall would be possible in a nonaxisymmetric gravitational potential (e.g., streaming along a bar). But since Seyferts do not seem to be preferentially barred galaxies (Heckman 1978; Simkin, $\mathrm{Su}$, and Schwarz 1980), this mechanism could not be generally relevant.

In view of the above arguments, together with the direct evidence for outflow that we observe in NGC $2992(\S \mathrm{V})$ and which is also seen in NGC 1068 (Walker 1968), we prefer the outflow to the infall interpretation of the line asymmetries.

In either case one would then expect the most asymmetric lines to occur in the dustiest NLRs. This does seem to be the case, as illustrated by the good correlation of the asymmetry index with the Balmer decrement.

The lack of any significant correlation between both the line widths and asymmetries, and the inclinations of the surrounding galaxies, argues that the flow orientation is not closely related to the angular momentum axis of the galaxy as a whole. It also provides further evidence that radial flow and not rotation is the principal line-broadening mechanism in the NLR.

The lack of a correlation between line width and nuclear continuum luminosity suggests that if the flow is driven by radiation pressure, then the NLR must adjust its structure to compensate for this greater luminosity (e.g., the characteristic size could increase $\propto L^{0.5}$ ). Alternatively, it may imply that the flow is not driven by radiation pressure. Since radiation pressure should work most effectively in the dustier NLRs, while we see no correlation between line width and either line asymme- 
try or $\mathrm{H} \alpha / \mathrm{H} \beta$, this mechanism might be regarded as unlikely on other grounds.

\section{b) Physical Conditions in the NLR}

Our observation that the apparent degree of ionization characterizing the NLR gas in class 1 Seyferts declines in the dustiest NLRs (Fig. 5) can be understood in several ways. First, it may be that the dust modifies the spectrum of ionizing photons as they travel from the nucleus to the NLR clouds. This could occur in such a way as to decrease the degree of ionization of the gas (e.g., the highest energy photons could be preferentially absorbed). A different possibility (cf. Pelat, Alloin, and Fosbury 1980) is that if there is an ionization gradient in the NLR - in the sense that the most highly ionized gas is located closest to the nucleus-then the greater the degree of dust extinction in the NLR, the more effectively the region of highly ionized gas will be hidden from our view. This could well produce the effect seen in Figure 5. The absence of a corresponding effect for the class 2 Seyferts must mean that the geometrical relationship between the gas, dust, and source of ionization is different from the simple pictures sketched above. This would concur with the conclusion drawn by Heckman and Balick (1979) that significant differences in the physical conditions in the NLR exist between the two Seyfert classes.

We have found no correlation between line width and either $n_{e}, T_{e}$, or the degree of ionization in NLR. This has an important consequence: the kinematical conditions in the NLR do not strongly affect the physical conditions and vice versa. Figure 7 reiterates this point, since there is no indication that class 1 and class 2 Seyferts define different relationships between radio power and line width, despite apparent differences in the nature of the NLR in the two classes. Even objects like M87 and 3C 236, in which the ionization mechanism is very different from that in Seyfert galaxies (cf. Ford and Butcher 1979; Heckman 1980b), appear to fall on the same relationship in Figure 7.

\section{c) NLRs and Radio Emission}

The close connection between the thermal and nonthermal regimes in Seyfert galaxies has been demonstrated on a variety of grounds (cf. Wilson and Willis 1980). In particular, we have shown (Fig. 7) that a good correlation exists between the NLR line width and the radio power of the kiloparsec-size "steep-spectrum core" radio source. We can ask what dependence between $P_{\text {Rad }}$ and $\Delta V$ would be predicted on the basis of some simple assumptions and compare the result with Figure 7. First, if the kinetic energy in the thermal gas $\left(\frac{1}{2} M V^{2}\right)$ is taken as being equal to the equipartion energy in the radio source $\left(E_{\mathrm{Eq}}\right)$, as seems to be roughly the case in
Seyfert galaxies (Wilson and Willis 1980), then, since

$$
P_{\mathrm{Rad}} \propto E_{\mathrm{Eq}}^{7 / 4}
$$

(from standard synchrotron theory) and

we get

$$
V^{2} \propto E_{\mathrm{Eq}},
$$

$$
P_{\mathrm{Rad}} \propto V^{3.5} \text {. }
$$

On the other hand, if we take the rate of kinetic energy flux through the NLR [e.g., $\frac{1}{2} M V^{2} /\left(R_{\mathrm{NLR}} / V\right)$ ] as being equal to the radio power (also approximately true according to Wilson and Willis), we would find

$$
P_{\mathrm{Rad}} \propto V^{3} .
$$

$P_{\mathrm{Rad}} \propto V^{3}$ or $V^{3.5}$ appears to yield a somewhat shallower relationship than that defined by Figure 7. Since most of the scatter in Figure 7 is intrinsic to the objects rather than due to measuring errors, only a substantial increase in the sample size can test the above simple models in a more critical way.

The direction of the causal link between $P_{\mathrm{Rad}}$ and line width is not yet obvious. For instance, the outflow of the NLR gas could power the radio source (the relativistic particles can be reaccelerated in shocks associated with the supersonic flow of the NLR gas). Conversely, the ejection of radio plasma from the inner nucleus could resupply the NLR with kinetic energy. Both of these possibilities have been described by Wilson and Willis (1980). Finally it is possible that rather than being linked in a direct, causal way the radio luminosity and the NLR kinematics are determined by the energetics of a common origin.

\section{CONCLUSIONS}

We can draw the following conclusions from our sample of 36 Seyferts and radio galaxies:

1. The majority of the [O III] profiles are markedly asymmetric with a significantly sharper falloff to the red than to the blue. This is in the opposite sense to the asymmetry which is preferentially observed in the wide permitted lines from the broad-line region (Osterbrock 1977).

2. The velocities derived for the [O III] lines are significantly negative with respect to the systemic velocities of the host galaxies.

3. There is an excellent agreement between the degree of asymmetry in the [O III] lines and the $\mathrm{H} \alpha / \mathrm{H} \beta$ intensity ratio.

4. The above results lead to the conclusion that the narrow-line region consists of dust and gas flowing radially (probably outward) with respect to the nucleus.

5. For nuclei having a broad-line region, the degree of profile asymmetry is inversely correlated with the $[\mathrm{O} \mathrm{III}] /[\mathrm{O} \mathrm{II}]$ and $[\mathrm{O} \mathrm{III}] /[\mathrm{O} \mathrm{I}]$ intensity ratios, indicating the influence of dust on the ionization structure of the 
NLR. The lack of a corresponding correlation in the nuclei that do not have broad-line regions may imply that in the latter case photoionization by the dilute ultraviolet continuum from the nucleus is not the dominant excitation mechanism for the NLR.

6. The relationship between the line widths and absolute radio luminosities for Seyfert 2 galaxies has been extended to the steep-spectrum, kiloparsec-sized, nuclear radio sources in Seyfert 1 and radio galaxies. This relationship demonstrates a close connection between the thermal and relativistic regimes in active nuclei.

7. The relative independence of the line width-radio luminosity relation on the type of nucleus involved, as well as the lack of any correlation between line width and $n_{e}, T_{e}$, or the characteristic ionization state, suggest that the kinematics and physical conditions in the NLRs are largely decoupled.

There are several lines of investigation that should be pursued to extend this work. Comparison of the line profiles of different ionization species can provide important information about the ionization structure of the NLRs. Theoretical modeling of the various effects (both static ionization and kinematic flow) are urgently required. Furthermore, the sample of profiles should be enlarged to include QSOs as well as additional Seyfert and radio galaxies, so that comparison of the profile parameters with additional optical, radio, and X-ray parameters can be made.

We are grateful to the staff of Kitt Peak, particularly Garth Illingworth and Doug McElroy, for their assistance with the observations and data reduction. We thank Professor Oort and Ger de Bruyn for their helpful critiques of an earlier version of this paper. We also thank Dan Weedman and Fred Feldman for interesting discussions concerning their related work. We acknowledge financial support provided by NATO Research grant 1828 .

\section{REFERENCES}

Adams, T. F. 1973, Ap. J., 179, 417. 1977, Ap. J. Suppl., 33, 19.

Baldwin, J. A. 1975, Ap.J., 201, 26.

Balick, B., and Heckman, T. 1979, A.J., 84, 302.

Bell, M. B., and Seaquist, E. R. 1980, Ap. J., 238, 818.

Boksenberg, A., Carswell, R. F., Allen, D. A., Fosbury, R. A. E., Penston, M. V., and Sargent, W. L. W. 1977, M.N.R.A.S., 178, 451.

Bosma, A., Ekers, R. D., and Lequeux, J. 1977, Astr. Ap., 57, 97.

Burbidge, E. M., Strittmatter, P. A., Smith, H. E., and Spinrad, H. 1972, Ap. J. (Letters), 178, L43.

Capriotti, E., Foltz, C., and Byard, P. 1979, Ap. J., 230, 681.

Costero, R., and Osterbrock, D. E. 1977, Ap. J., 211, 675.

Davidson, K., and Kinman, T. D. 1978, Ap. J., 225, 776.

de Bruyn, A. G. 1980, private communication.

de Bruyn, A. G., and Wilson, A. S. 1976, Astr. Ap., 53, 93. 1978, Astr. Ap., 64, 433.

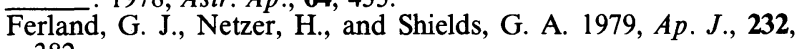
382.

Fomalont, E. B., Miley, G. K., and Bridle, A. H. 1979, Astr. Ap., 76, 106.

Ford, H. C., and Butcher, H. 1979, Ap. J. Suppl., 41, 147.

Fricke, K. J., and Reinhardt, M. 1974, Astr. Ap., 37, 349.

Glaspey, J. W., Eilek, J. A., Fahlman, G. G., and Auman, J. R. 1976, Ap. J., 203, 335.

Glaspey, J. W., Walker, G. A. H., and Stockton, A. 1976, Ap. J., 210, 27.

Graham, I. 1970, M.N.R.A.S., 149, 319.

Grandi, S. A. 1977, $A p$. J., 215, 446 . 1978, Ap. J., 221, 501

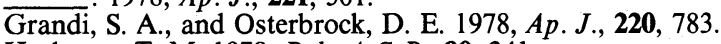

Heckman, T. M. 1978, Pub. A.S.P., 90, 241. . 1980 a, Astr. Ap., 87, 142. . 1980 b, Astr. Ap., 87, 152.

Heckman, T. M., and Balick, B. 1979, Astr. Ap., 79, 350. 1981, in preparation.

Heckman, T. M., Balick, B., and Crane, P. C. 1980, Astr. Ap. Suppl., 40, 295.

Heckman, T. M., Balick, B., and Sullivan, W. T. 1978, Ap. J., 224, 745.

Keel, W. C. 1980, A.J., 85, 198.
Kellermann, K. I., Pauliny-Toth, I. I. K., and Williams, P. J. S. 1969, Ap. J., 157, 1.

Kent. S. M., and Sargent, W. L. W. 1979, Ap. J., 230, 667.

Koski, A. T. 1978, Ap. J., 223, 56.

Maza, J. 1979, Ph.D. thesis, University of Toronto.

Miley, G. 1980, Ann. Rev. Astr. Ap., 18, 165.

Miley, G. K., and Osterbrock, D. E. 1979, Pub. A.S.P., 91, 257.

Miley, G. K., and Perola, G. C. 1975, Astr. Ap., 45, 223.

Neugebauer, G., et al. 1980, Ap. J., 238, 502.

Osmer, P. S., Smith, M. G., and Weedman, D. W. 1974, Ap. J., 192, 279.

Osterbrock, D. E. 1977, Ap. J., 215, 733.

Osterbrock, D. E., Koski, A. T., and Phillips, M. M. 1976, Ap.J., 206, 898.

Pelat, D., and Alloin, D. 1980, Astr. Ap., 81, 172.

Pelat, D., Alloin, D., and Fosbury, R. A. E. 1980, ESO Preprint No. 98.

Penston, M. V., and Fosbury, R. A. E. 1978, M.N.R.A.S., 183, 479.

Phillips, M. M. 1976, Ap. J., 208, 37.

Rieke, G. H. 1978, Ap. J., 226, 550.

Rubin, V. C., and Ford, W. K. 1968, Ap. J., 154, 431.

Rubin, V. C., Ford, W. K., Peterson, C. J., and Oort, J. H. 1977, Ap. J., 211, 693.

Rubin, V. C., Thonnard, N., and Ford, W. K. 1975, Ap.J., $199,31$.

Schechter, P. L., and Gunn, J. E. 1979, Ap. J., 229, 472.

Schmidt, G. D., and Miller, J. M. 1980, Ap. J., 240, 759.

Simkin, S. M., Su, H. J., and Schwarz, M. P. 1980, Ap. J., 237, 404.

Stockton, A. N. 1968, A.J., 73, 887.

Thompson, I., Landstreet, J. D., Stockman, H. S., Angel, J. R. P., and Beaver, E. A. 1980, M.N.R.A.S., 192, 53.

Ulrich, M.-H. 1973, Ap.J., 181, 51.

Walker, M. F. 1968, Ap. J., 151, 71

Ward, M. J., Wilson, A. S. Penston, M. V., Elvis, M., Maccacaro, T., and Tritton, K. P. 1978, Ap.J., 223, 788.

Weedman, D. W. 1977, Ann. Rev. Astr. Ap., 15, 69.

Wehinger, P. A., and Wyckoff, S. 1977, M.N.R.A.S., 181, 211.

Wilson, A. S. 1979, Proc. Roy. Soc. London, 366, 461.

Wilson, A. S., and Penston, M. V. 1979, Ap.J., 232, 389.

Wilson, A. S., and Willis, A. G. 1980, preprint.

Harvey R. Butcher and Wil J. M. van Breugel: Kitt Peak National Observatory, P.O. Box 26732, Tucson, AZ 85726

Timothy M. HeckMan: Steward Observatory, University of Arizona, Tucson, AZ 85721

GeORGe K. MiLeY: Sterrewacht Leiden, Postbus 9513, 2300 RA Leiden, The Netherlands 